\title{
A deep cluster survey in Chandra archival data. First results
}

\author{
W. Boschin ${ }^{\star}$ \\ Dipartimento di Astronomia, Università degli Studi di Trieste, via Tiepolo 11, 34131, Trieste, Italy \\ Received 30 July 2002 / Accepted 19 September 2002

\begin{abstract}
I present the first results of a search for clusters of galaxies in Chandra ACIS pointed observations at high galactic latitude with exposure times larger than $10 \mathrm{ks}$. The survey is being carried out using the Voronoi Tessellation and Percolation technique, which is particularly suited for the detection and accurate quantification of extended and/or low surface brightness emission in X-ray imaging observations. A new catalogue of 36 cluster candidates has been created from $5.55 \mathrm{deg}^{2}$ of surveyed area. Five of these candidates have already been associated to visible enhancements of the projected galaxy distribution in low deepness DSS-II fields and are probably low-to moderate redshift systems. Three of the candidates have been identified in previous ROSAT-based surveys. I show that a significative fraction (30-40\%) of the candidate clusters are probably intermediate to high redshift systems. In this paper I publish the catalogue of these first candidate clusters. I also derive the number counts of clusters and compare it with the results of deep ROSAT-based cluster surveys.
\end{abstract}

Key words. cosmology: large-scale structure of Universe - galaxies: clusters: general - X-rays: galaxies: clusters

\section{Introduction}

Clusters of galaxies are very important objects in astrophysics. Observations of such systems at different redshifts can be used, for instance, to explore galaxy evolution in very dense environments (e.g. Dressler et al. 1997; Jorgensen et al. 1997; Kelson et al. 1997; Ellingson et al. 2001; Nelson et al. 2001; van Dokkum \& Franx 2001). Clusters are also crucial probes of mass distribution at intermediate and large scales (e.g. Nichol et al. 1992; Collins et al. 2000; Schuecker et al. 2001; Gonzalez et al. 2002). Moreover, in the scenario of hierarchical structure formation, the estimate of cluster abundance as a function of their mass and redshift plays a fundamental role in constraining the cosmological parameters $\Omega_{\mathrm{m}}$ and $\sigma_{8}$ (e.g. Bahcall \& Cen 1993; Girardi et al. 1998; Borgani et al. 2001; Reiprich \& Böhringer 2002). Following a different approach, observations of the Sunyaev-Zeldovich effect (Sunyaev \& Zeldovich 1972) in a large sample of distant clusters can also be used for a direct measurement of their distances, and can thus provide estimates of the Hubble's constant (Mauskopf et al. 2000; Reese et al. 2000).

In any case, all these studies require large samples of clusters in a wide range of redshift, from $z=0$ to $z=1$ and beyond. In particular, nowadays much interest is given to the search for medium-high redshift clusters, whose physical and statistical properties need a big effort of investigation. Up until the present, the largest samples of distant clusters have resulted from optical/NIR and X-ray sky surveys. Optical/NIR cluster surveys consist in searching for enhancements in the surface

\footnotetext{
* e-mail: boschin@ts.astro.it
}

density of galaxies (e.g. Postman et al. 1996; Nonino et al. 1999). This classical technique suffers seriously from projection effects, sometimes leading to false identifications, particularly for poor clusters at high redshift, where the contrast with the background galaxy surface density is low. Another problem of these surveys is that the cluster galaxy richness has a poor correlation with mass (e.g. Borgani \& Guzzo 2001). This does not assure that the selected sample contains all clusters within a given volume and above a given mass, since the cluster selection function is only loosely related to this fundamental parameter, which is what model predictions are based upon. Therefore it is difficult to use optically/NIR selected clusters for cosmological studies. The advantage of optical surveys is the availability of a large number of ground telescopes with high quantum efficiency detectors. This makes completing wide area surveys an easy and relatively quick task. In fact, recent deep optical/NIR surveys (e.g. the Las Campanas Distant Cluster Survey) are now reaching areas in excess of $100 \mathrm{deg}^{2}$ (Gonzalez et al. 2001; Nelson et al. 2002) enabling the discovery of the richest clusters at $z \sim 1$ and the study of the evolution of the moments of the cluster galaxy distribution.

Instead, X-ray cluster surveys consist in detecting the X-ray emission from the hot gas which fills the space between cluster galaxies. Even if large area X-ray surveys are time intensive, their big advantage is that clusters look sharper in the X-ray than in the optical sky. In fact, while the optical emission is approximately only linearly dependent on the number of cluster galaxies, the X-ray emission is proportional to the square of the local gas density. Moreover, the detection of the intracluster medium is an unambigous indicator of a real cluster. 
For this reason the problem of spurious detection is much less severe. But the best feature of X-ray surveys is that their selection function can be determined to high accuracy knowing the properties of the telescope used. Moreover, since X-ray luminosity is a good indicator of the total cluster mass (Reiprich \& Böhringer 1999), we can safely make cosmological predictions for clusters of a given mass and sensibly compare them with observed clusters of a given luminosity. In synthesis, X-ray surveys are much easier to use than optical/NIR ones as cosmological probes to address global cluster properties and not only for studying single, yet interesting, objects.

In the past decade these good qualities encouraged the compilation of many X-ray cluster surveys mainly based on ROSAT data (e.g. Rosati et al. 1995, 1998; Scharf et al. 1997; Vikhlinin et al. 1998). All these surveys gave important contributions in determining the X-ray cluster number density and luminosity function at lower and lower flux limits.

In this paper, I present the first results of a search for galaxy clusters in deep (exposure time $T>10 \mathrm{ks}$ ) Chandra ACIS pointed observations at high Galactic latitude. The Chandra's high spatial resolution is the strong point of a survey based on this telescope, since it is essential to efficiently separate between pointlike and extended sources and, in particular, to reduce the problem of the contamination of distant clusters by active galactic nuclei. The survey is being carried out using the Voronoi Tessellation and Percolation technique (VTP, Ebeling \& Wiedenmann 1993). This approach is completely non parametric, i.e. it is equally sensitive to spherically simmetric structures and to irregular ones, and shows its effectiveness to detect low surface brightness sources with greater sensitivity when compared with standard detection methods (Scharf et al. 1997).

This paper is arranged as follows: in Sect. 2 I present a brief overview of the Chandra selected pointings and the data reduction; in Sect. 3 I describe the source detection method and the steps of the construction of the catalogue. In Sect. 4 I present the calibration of the survey sky coverage. Section 5 presents an estimate of the redshift distribution of the detected candidates and a measure of the sky volume probed by the survey. In Sect. 6 and 7, I present the $\log N-\log S$ relation and the catalogue of detected candidates. Finally, in Sect. 8, I summarize the main results and give my conclusions.

Computations throughout the paper use $\Omega_{\mathrm{m}}=0.3, \Omega_{\Lambda}=$ 0.7. The Hubble constant is $H_{0}=75 \mathrm{~km} \mathrm{~s}^{-1} \mathrm{Mpc}^{-1}$, unless otherwise stated. All X-ray luminosities and fluxes are reported in the $0.5-2.0 \mathrm{keV}$ energy band.

\section{Observations and data analysis}

The data used in this paper are 81 Chandra ACIS pointed observations downloaded from the public archive. Selected observations have exposure times larger than $10 \mathrm{ks}$ (see Fig. 1) and absolute galactic latitude larger than $20 \mathrm{deg}$. This skyselection criterium minimizes the effects of absorption of extragalactic X-rays from the galactic neutral hydrogen concentrated around the Milky Way plane. In general, the galactic hydrogen column density along the line of sight of the selected pointings is $n_{\mathrm{H}}<10^{21} \mathrm{~cm}^{-2}$ (see Fig. 2). I preferentially avoid pointings whose targets are bright extended sources

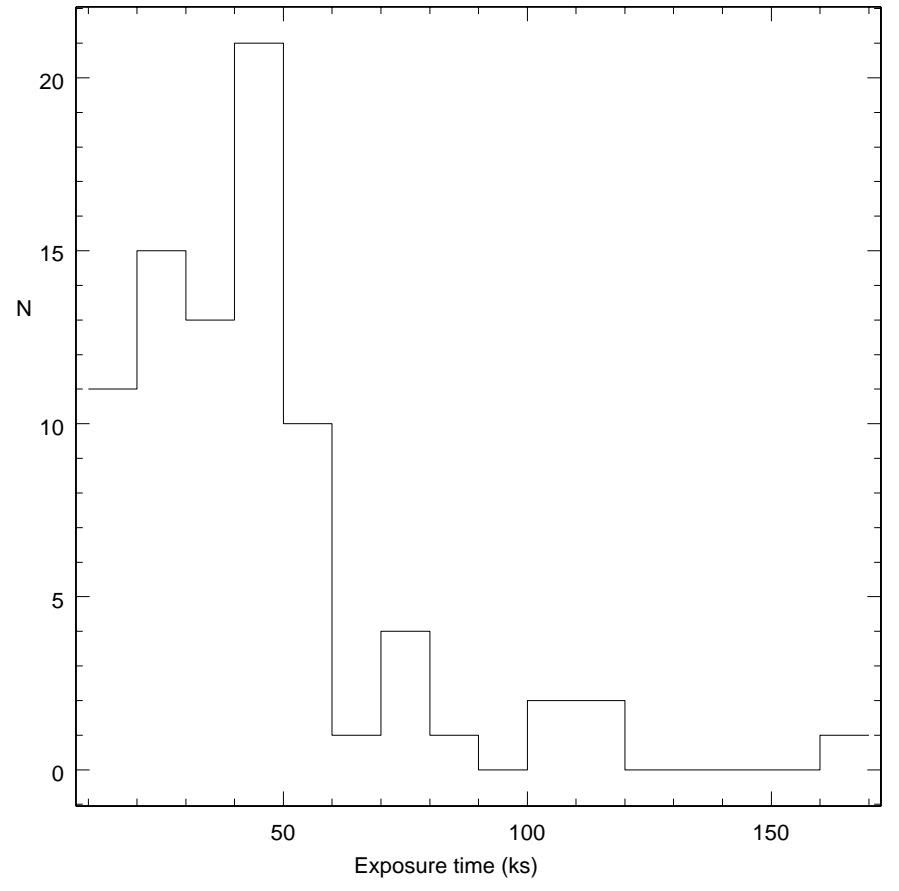

Fig. 1. Histogram of the exposure times for the 81 selected Chandra ACIS pointings.

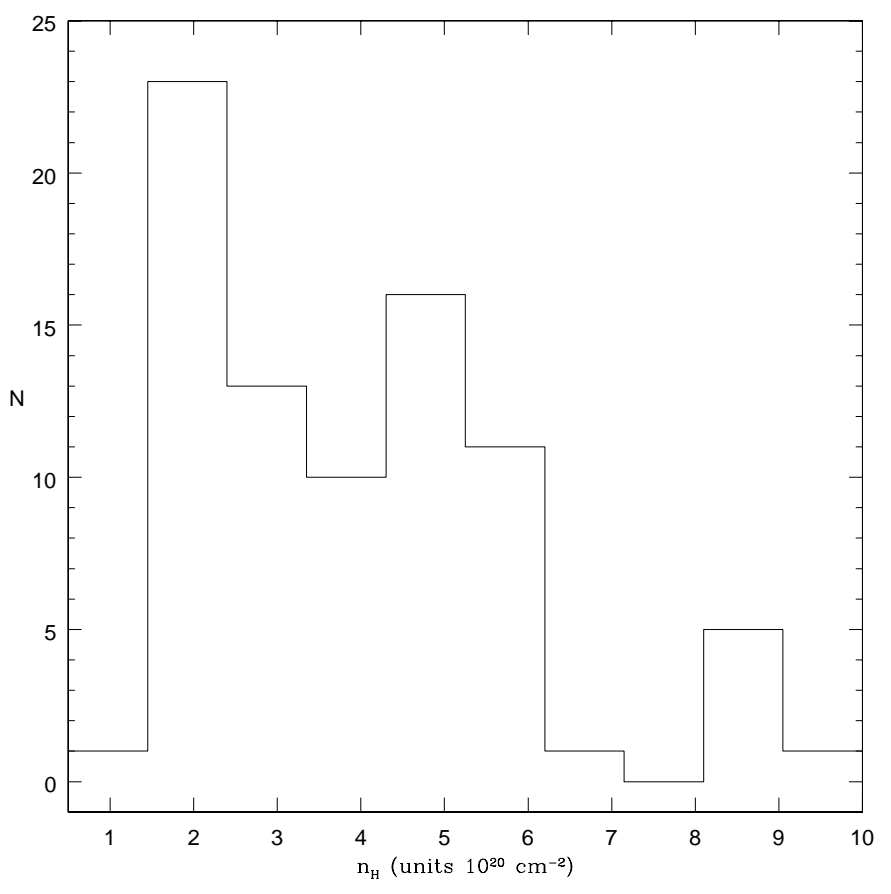

Fig. 2. Histogram of the galactic neutral hydrogen column density along the line of sight of the 81 selected Chandra ACIS pointings.

(typically nearby clusters and galaxies), since they dominate the field of view and make it impossible the detection of other objects. This also ensures that the cluster selection function is not significantly affected by the known angular correlation between galaxy clusters. In Fig. 3, the positions of the 81 selected Chandra fields are plotted in Galactic Coordinates. The size of the points is proportional to the exposure times, which ranges from 11 to $168 \mathrm{ks}$. 


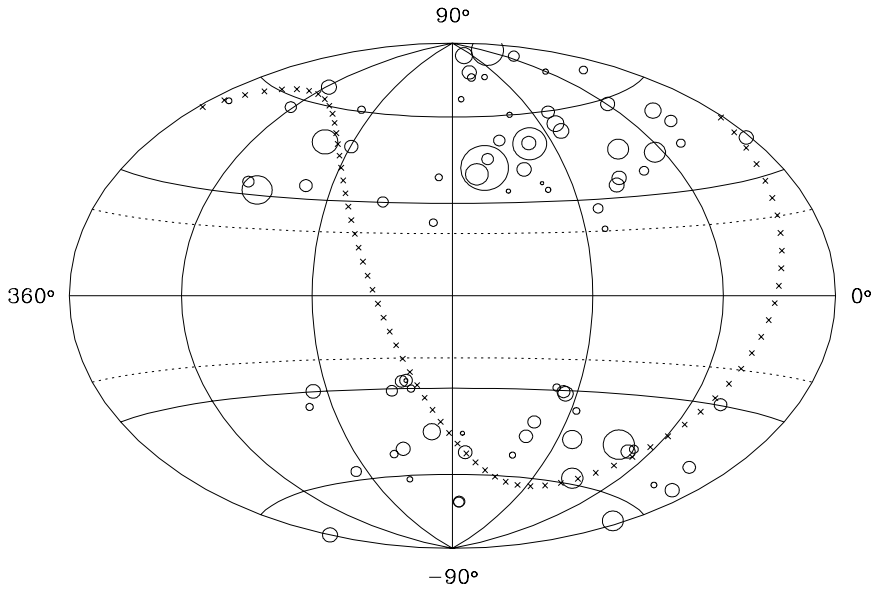

Fig. 3. The 81 Chandra ACIS pointings selected for this survey (Aitoff projection in galactic coordinates). The sizes of points are proportional to the exposure time. Dotted horizontal lines delimit $|b|=20^{\circ}$; hatched line is the celestial equator $\left(\delta=0^{\circ}\right)$.

The Chandra ACIS is a scientific instrument made of two blocks. One, ACIS-Imager (ACIS-I), is a square matrix of $41024 \times 1024$ pixels front-illuminated (FI) CCDs (chips I0, I1, I2 and I3) covering a field of view of about 17 by 17 arcmin and is used for imaging observations. The other one, ACISSpectrometer (ACIS-S), is an array of $61024 \times 1024$ pixels CCDs (4 front-illuminated, 2 back-illuminated (BI)) covering a field of view of about 50 by 8 arcmin and is used both for imaging observations and, mainly, for spectroscopical observations with gratings. In general, during an observation ACIS can operate simultaneously both with ACIS-I CCDs and with ACIS-S CCDs, for a maximum of 6 active CCDs. I consider here both images taken by ACIS-I chips (chips I0, I1, I2 and I3), and ACIS-S imaging observations (aimpoint on S3) taken by chips S2, S3, I2 and I3. In ACIS-S observations I take into consideration only the FI chips (S2, I2 and I3). I choose to search for X-ray sources only in these chips since they manifest a similar photon background. This is important for a correct use of the source detection algorithm (see Sect. 3). Moreover, in the selected ACIS-S pointings the S3 chip is often completely filled by target photons and, therefore, unusable for the search of other sources.

Before Chandra observations be used to search for sources, they need some reduction. I perform the data reduction using the dedicated software CIAO (Chandra Interactive Analysis of Observations, Fruscione \& Siemiginowska 2000). For each pointing, first I filter the preprocessed event files selecting only events with a status equal to zero and with ASCA grades 0, 2, 3, 4 and 6 . This particular grade selection appears to optimize the signal-to-background ratio of sources (Chandra X-ray Center Team, 2001). Then, I clean bad offsets intervals and examine the data on a chip by chip basis. Since for each photon event the energy is known, I select all events with energy between 0.3 and $10 \mathrm{keV}$. This is the band in which the energy scale of ACIS CCDs is calibrated. For each chip I filter out bad columns and pixels and remove times when the count rate exceeded three standard deviations from the mean count rate per $3.3 \mathrm{~s}$ frame time intervals. In fact, the background is generally quite stable during an observation. Nevertheless, occasionally there are significant variations, especially in the BI chips but also in FI ones. Since for each photon event the occurance time is known, I am able to filter out high background intervals. I then clean each chip for flickering pixels (pixels showing unstable states of dark current signature), i.e. I remove times where a pixel had events in two sequential $3.3 \mathrm{~s}$ intervals. At the end, I remain with a clean image containing photon events with energies between 0.3 and $10 \mathrm{keV}$.

\section{Source detection and building of the catalogue}

The first step in the detection process consists in the choice of an energy band from which extract photons to pass to the detection algorithm. The output of the reduction step is a photon event file containing photons with energies in a wide energy range $(0.3-10 \mathrm{keV})$. However, it is not convenient to use the whole band in order to detect sources. In fact, unless we are dealing with nearby massive systems, clusters emit generally very few photons in the hard energy band (with energies larger than 4-5 keV), therefore in order to maximize their contrast with respect to the X-ray background and enhance the detection probability, it is necessary to work only with the softest X-rays. In particular, Scharf (2002) calculates the maximum, or optimal, signal-to-noise energy band for galaxy system X-ray emission detected by the Chandra observatory. He finds that the "classical" ROSAT-like $0.5-2.0 \mathrm{keV}$ band is close to optimal for clusters in a wide range of intracluster medium (ICM) temperature (in particular for $T>2 \mathrm{keV}$ ) and redshift $z \leq 1$. Based on these considerations, for the detection of sources I choose to consider only photons in the energy range $0.5-2.0 \mathrm{keV}$.

As a detection algorithm I use the Voronoi Tessellation and Percolation technique (hereafter VTP). The VTP method of Ebeling (1993, see also Ebeling \& Wiedenmann 1993) is a general method for the detection of non Poissonian structure in a distribution of points. By definition, a Voronoi tessellation on a two-dimensional distribution of points (called nuclei) is a unique plane partition into convex cells, each of them containing one, and only one, nucleus together with the set of points which are closer to that nucleus than to any other one (Voronoi 1908). In X-ray observations, any nucleus is a detector pixel where at least one photon count occurs. To each of these pixels, VTP associates a cell polygon whose sides form the perpendicular bisectors of the non-crossing vectors joining the nearest neighbor photon pixels. Since most cells contain only one photon, the surface brightness associated with this photon equals the inverse of the product of cell area and local exposure time. Multiple counts pixels are rare and associated only to the brightest sources. These pixels are flagged and used in the percolation algorithm described below.

In Fig. 4, I show the Voronoi tessellation of a typical Chandra ACIS-I photon distribution. The sources in this field are apparent to the eye as clusters of small cells. After the implementation of the Voronoi tessellation, the cumulative distribution of the inverse areas of the photon cells is computed and compared with that expected from a random (Poisson) distribution (Kiang 1966), and a cutoff can be determined that defines the global background count for that field. A spatial 


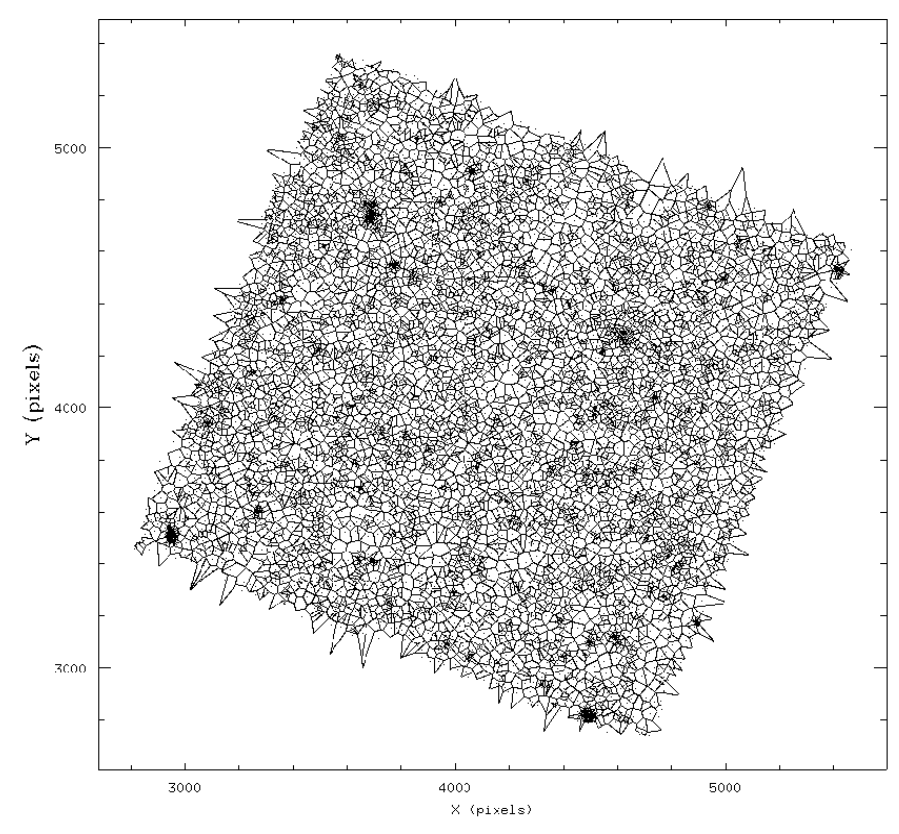

Fig. 4. Voronoi tessellation of a typical Chandra ACIS-I photon distribution. Photons are shown as dots and sources are immediately apparent to the eye as clusters of small tessellation cells.

percolation algorithm is then run on the cells with area smaller than a given threshold above the background, grouping them according to the excess above the background density and forming sources. For a better background estimation the latter two steps are performed iteratively so that, as sources are detected, the background estimate is revised and the source groupings are redetermined. Finally, for each source I obtain a series of parameters: the source position (computed as a weighted sum over photons), the detected source count rate, the detected source area, the minimal and maxima moments of inertia of the photon distribution, an estimate of the background count, and the probability of the source being a statistical fluctuation. Moreover, the full set of photons associated with each source is stored.

The VTP procedure is implemented on a pipeline composed by the CIAO task vtpdetect and a $\mathrm{C}$ code representing an adaptation of the code developed by Ramella et al. (2001) to detect galaxy clusters in optical photometric surveys.

\subsection{Source deblending}

As a first step, VTP is run on each field using as a surface brightness threshold the computed value of the background surface brightness. However, in general it is not convenient to run VTP with a unique surface brightness threshold. In fact, VTP has, by design, a potentially serious drawback: it is unable to separate very close pairs of sources, i.e, it inevitably produces "blends" as soon as it finds the high-flux zones of two emission regions to be touching one another. In order to solve this problem, Scharf et al. (1997) show that a practical solution is to run VTP using different surface brightness thresholds. In this way, it is also possible to reduce the uncertainties in source identification resulting from positive background fluctuations that

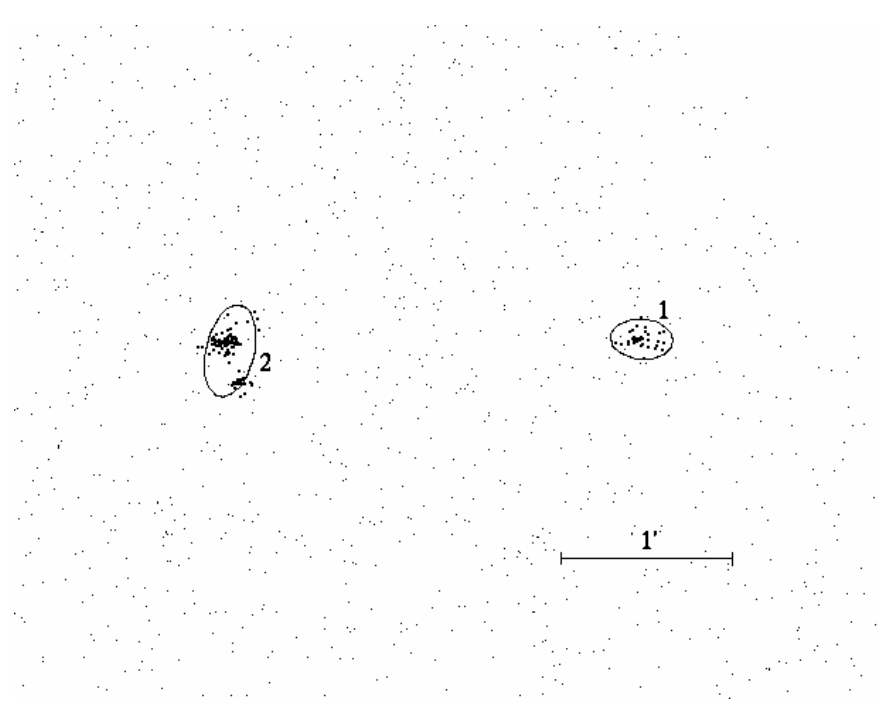

Fig. 5. Source photons and sources identified by VTP at threshold 1.0 in a portion of an ACIS-I field. Thick points indicate VTP source photons, sources are labeled numerically. Note that source 2 is a clear blend.

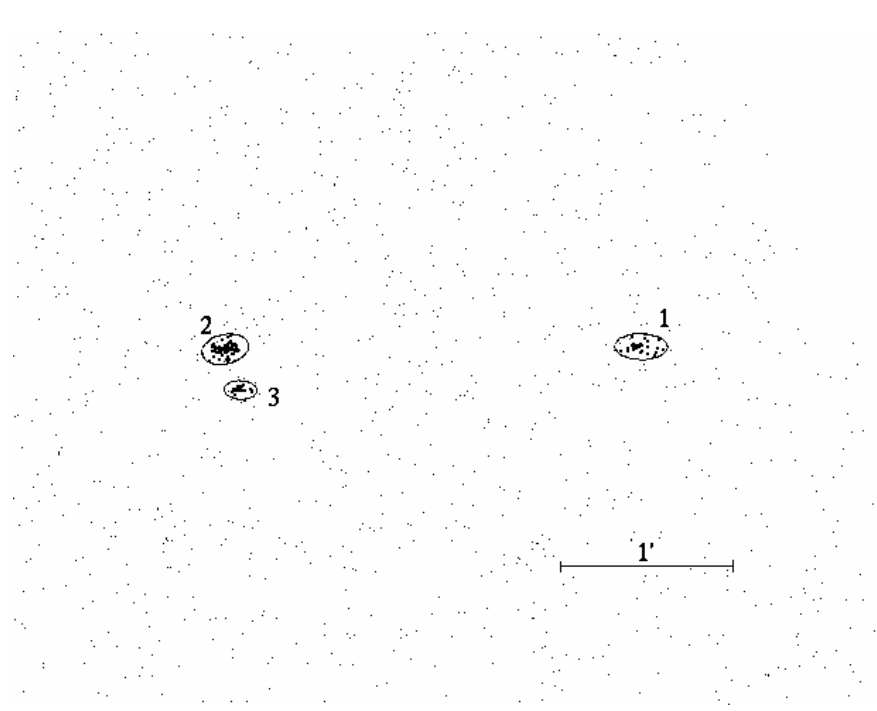

Fig. 6. Source photons and sources identified by VTP at threshold 1.7 in the field shown in Fig. 5. Note that source 2 at threshold 1.0 has now been resolved into sources 2 and 3 .

become grouped with source photons. I run VTP three times with three different thresholds (denoted as factors of the background surface brightness): 1.0 (the first step), 1.3 and 1.7. Then, I select the sources matching the following criteria:

- Sources at threshold 1.0, which do not deblend at higher thresholds, and with an observed count rate greater than $2 \times 10^{-4}$ counts $\mathrm{s}^{-1}$ (corresponding to an observed flux limit of $\left.\sim 1 \times 10^{-15} \mathrm{erg} \mathrm{s}^{-1} \mathrm{~cm}^{-2}\right)$;

- Sources matching the above count rate criteria at threshold 1.3 that were parts of blends at 1.0 but do not deblend at threshold 1.7;

- Sources matching the position and count rate criteria at threshold 1.7 that were parts of blends at threshold 1.3.

This deblending procedure provides a list of candidates that is used in a visual inspection of each field. In total, I find 
3340 X-ray sources in the 81 selected fields at the threshold 1.0. Raising the threshold level, the number of detected sources at thresholds 1.3 and 1.7 is, respectively, $94 \%$ and $84 \%$ of the total number detected at the lowest threshold. 65 sources $(2 \%)$ are deblended with the described procedure. For some individual sources, it was necessary to change by hand the detection threshold in order to exclude photons that were clearly positive noise and were included in the objects detected at threshold 1.0. These spurious photons clearly act to bias the area estimate of the source. In general, increasing the threshold removes the noise and typically only removes less than $10 \%-20 \%$ of the source photons. Moreover, these photons will be recovered in the flux correction step described below. The threshold used for each source is recorded and used in the correction from detected to total flux, as detailed in Sect. 3.3.

Figures 5 and 6 demonstrate the differences between the results for the lowest and highest threshold for a portion of an ACIS-I field. Photons identified as belonging to sources by VTP are plotted in heavy type. The source numbered 2 in Fig. 5 is a clear blend of two pointlike sources when observed at the lowest threshold. In Fig. 6, these two sources are deblended at the higher (1.7) threshold.

It is clear that, in principle, there may be cases of real physical systems containing substructures that become deblended. However, a visual inspection of the 81 fields does not reveal any case in which deblending had split up what was probably a single extended object into separate components. By visual inspection I also reject a set of 102 sources which appear as border effects or artifacts due to strong X-ray sources contained in the selected fields.

\subsection{Separation between pointlike and extended sources}

Once the deblending process is completed, the next step consists in separating between pointlike and extended sources. Really, the very small value of the PSF radius (less than $0.5 \operatorname{arcsec}$ at the aimpoint), makes this operation quite an easy task when considering on-axis sources. Nevertheless, a smart procedure is necessary when considering off-axis sources, expecially where the PSF radius is larger than about 10 arcsec. This is expecially true for sources located in chips I2 and I3 during ACIS-S observations. In order to build such a procedure, assumptions must be made about the nature of the sources. In particular, I assume that sources are either intrinsically extended or pointlike. In modeling a pointlike source I assume the PSF is Gaussian. The VTP algorithm returns estimates of the mean surface brightness of the background, $\sigma_{\mathrm{bkg}}$, a background corrected estimate $s$ of the observed count rate for each detected source, and the area of the source above the surface brightness threshold. From the area, I obtain an area equivalent source radius defined as $r_{\mathrm{VTP}} \equiv\left(A_{\mathrm{VTP}} / \pi\right)^{1 / 2}$. Supposing now the source is pointlike, the detected and background corrected count rate $s$ can be written as

$$
s=s\left(r_{\mathrm{VTP}}\right)=2 \pi \int_{0}^{r_{\mathrm{VTP}}} \sigma_{\mathrm{PSF}}(r) r \mathrm{~d} r,
$$

where $\sigma_{\mathrm{PSF}}(r)=\sigma_{0} \tilde{\sigma}_{\mathrm{PSF}}(r)$ is the assumed surface brightness profile for a Gaussian PSF, with $\tilde{\sigma}_{\mathrm{PSF}}(r)=\exp \left(-r^{2} / 2 \rho^{2}\right) . \rho$ is the width of the PSF. Besides Eq. (1), according to Ebeling et al. (1996), I need a second equation specifying how close to the background the surface brightness of the outermost regions of a VTP detection is:

$\sigma_{\mathrm{PSF}}\left(r_{\mathrm{VTP}}\right)=\sigma_{0} \tilde{\sigma}_{\mathrm{PSF}}\left(r_{\mathrm{VTP}}\right)=(f-1) \sigma_{\mathrm{bkg}}$.

Here $f$ is the normalized distance of the lowest surface brightness region to the background level. This parameter is returned by VTP for each source. Combining Eqs. (1) and (2) it is now possible to eliminate $\sigma_{0}$ and obtain an equation in the unknown $\rho$ which can be solved. I stress that at no stage of this procedure the model profile from Eq. (1) is actually fitted to the observed surface brightness distribution. Although I do assume a specific model (Gaussian in this case), it is only its integral properties that enter; thus, even if the ACIS PSF profile is not exactly a Gaussian, deviations of the true distribution from the assumed model have much less effect on the results than they do for the fitting procedures employed by conventional detection algorithms. However, the lack of any radial fitting also entails that the value for the source width $\rho$ determined in this process cannot be expected to be as accurate as what might be obtained in a detailed imaging analysis of pointed data.

Now, the estimated $\rho$ of the detected sources can be compared with the best-fit PSF $\rho$ as a function of the off-axis angle in order to discriminate between pointlike and extended sources. Of course, what happens is that extended sources will have estimated $\rho$ larger than the expected value for a pointlike source. In particular, the lower limits of $\rho$ for a source to be considered as extended are computed by building Monte Carlo simulations of ACIS fields. Sources are simulated with pointlike surface brightness, convolved with the instrumental PSF (for a nominal photon energy of $1.5 \mathrm{keV}$ ) and added to a representative background of Poissonian noise. For this purpose I use the package MARX (e.g. Wise 1997), a set of executables designed to be run in sequence to produce a Chandra/ACIS simulation. In particular, I simulate more than 500 pointlike sources with different count rates and signal-to-noise ratios $S N R$ (as defined in Sect. 5) and located at different off-axis angles. Figures 7-9 show the estimated $\rho$ for all the simulated pointlike sources vs. off-axis angles in the $S N R$ bins $>10,6-10$ and $<6$ respectively. For each figure, the solid line is a polynomial model fit to all data points, while the dotted line is a 99.9\% confidence level line derived from the $\rho$ residuals of the PSF best-fit model. For each source, the errorbars in the $\rho$ estimate are $1 \sigma$ errors derived from the fit. A source is considered as extended if its $\rho$ is larger than the PSF $\rho$ at the $99.9 \%$ confidence level in the corresponding $S N R$ range. In total, from the 81 selected fields I classify 51 extended sources.

\subsection{Source count rates correction for missing flux}

The presence of an X-ray background always limits the emission directly detectable by any source detection algorithm to some fraction of the total source flux. Therefore, the raw VTP count rates of the detected sources have to be corrected for low surface brightness emission in the far wings of the source 


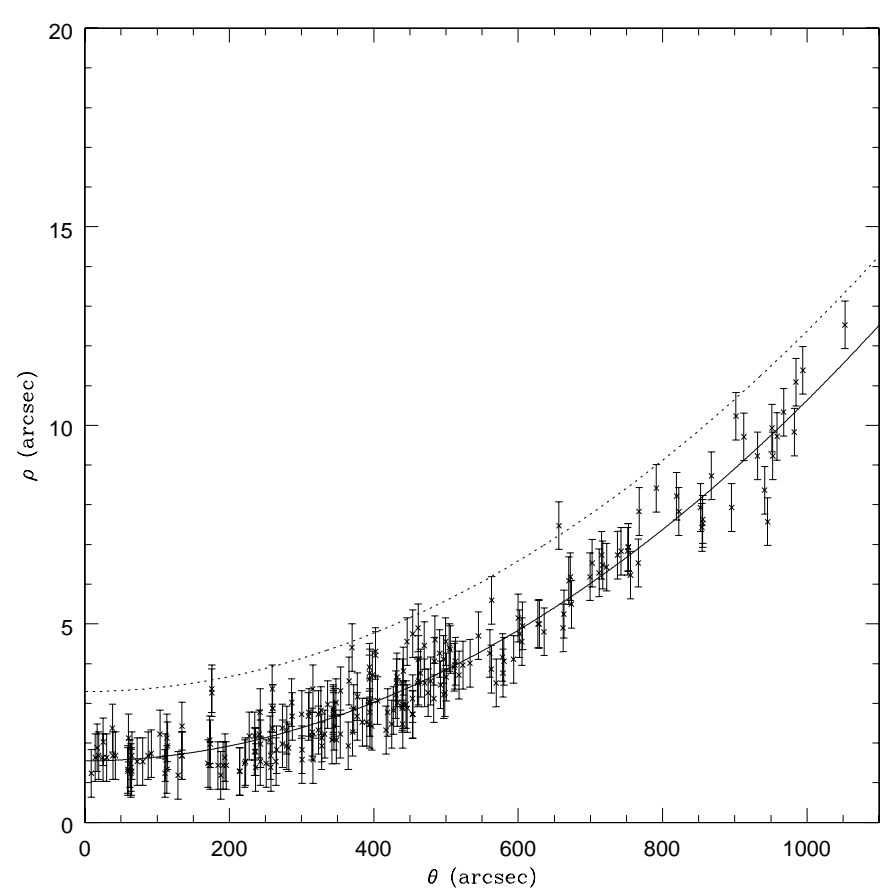

Fig. 7. Estimated $\rho$ and $1 \sigma$ errors for a set of pointlike simulated sources with $S N R>10$ vs. off-axis angle. The solid line is a polynomial fit to all data points. The dotted line is a $99.9 \%$ confidence level line derived from the $\rho$ residuals of the PSF best-fit model.

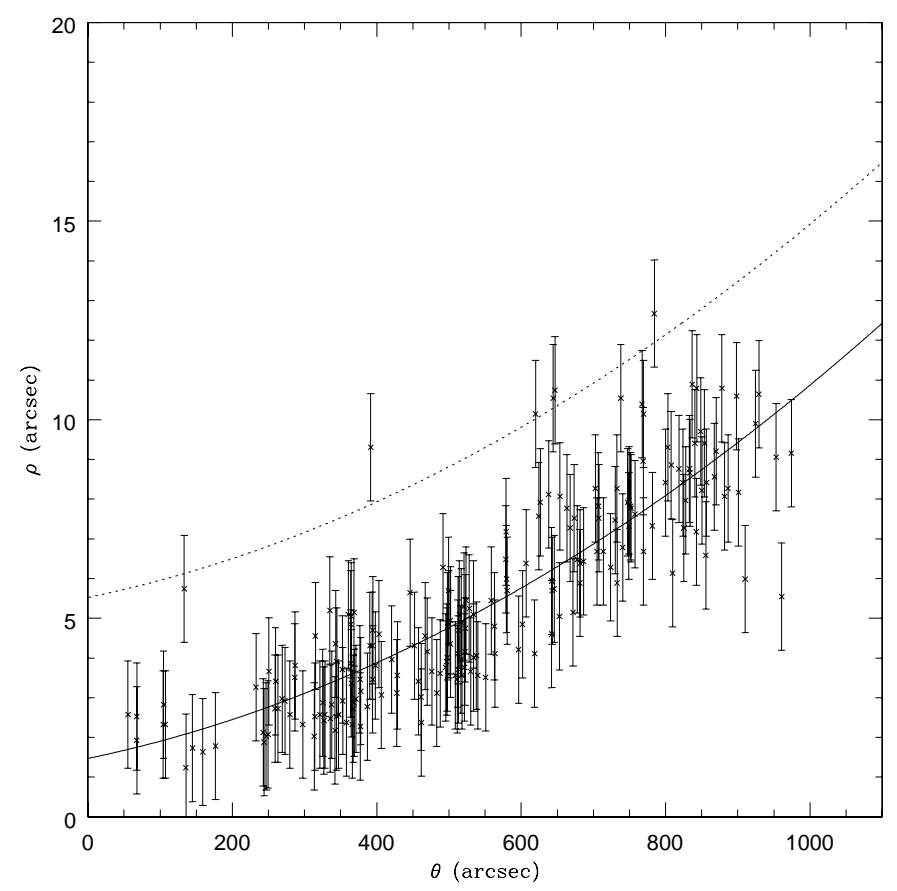

Fig. 8. Estimated $\rho$ and $1 \sigma$ errors for a set of pointlike simulated sources with $6<S N R<10$ vs. off-axis angle. The solid line is a polynomial fit to all data points. The dotted line is a $99.9 \%$ confidence level line derived from the $\rho$ residuals of the PSF best-fit model.

that has not been directly detected. This is particularly important for extended sources. In order to perform this correction I have to assume again a surface brightness profile for the detected sources. In particular, for the extended sources I adopt the King's approximation to the density profile of an

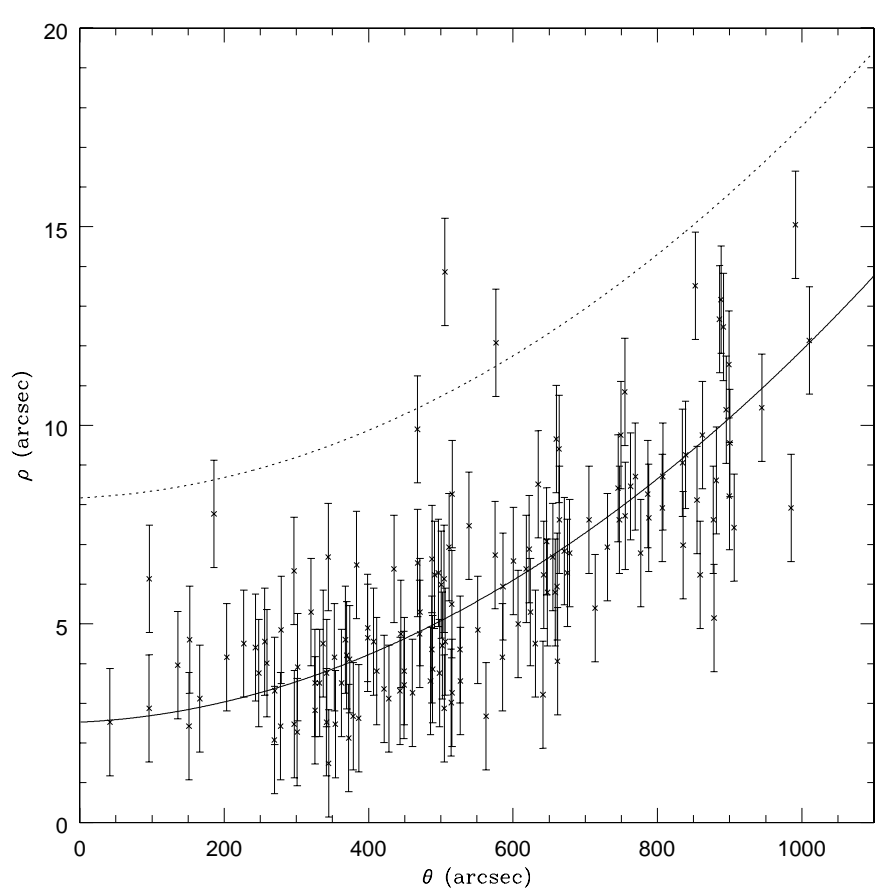

Fig. 9. Estimated $\rho$ and $1 \sigma$ errors for a set of pointlike simulated sources with $S N R<6$ vs. off-axis angle. The solid line is a polynomial fit to all data points. The dotted line is a $99.9 \%$ confidence level line derived from the $\rho$ residuals of the PSF best-fit model.

isothermal sphere (King 1962) and assume a source profile of the form (Cavaliere \& Fusco Femiano 1976)

$\sigma_{\mathrm{K}}(r)=\sigma_{0}\left[1+\left(r / r_{\mathrm{c}}\right)^{2}\right]^{-3 \beta+1 / 2}$,

where $\sigma_{\mathrm{K}}(r)$ is the projected surface brightness as a function of radius, $\sigma_{0}$ is the central surface brightness and the $r_{\mathrm{c}}$ is the core radius. I also assume $\beta=2 / 3$. This value of $\beta$ is a $\operatorname{good}$ approximation for nearby clusters (Jones \& Forman 1984). At medium-high redshifts, clusters are characterized by values of $\beta$ in the range $0.5-0.8$ (e.g. Arnaud et al. 2002; Holden et al. 2002; Pointecouteau et al. 2002), thus $\beta=2 / 3$ is intermediate between these values. Now, from the knowledge of background surface brightness and of the VTP source characteristics I can numerically compute $\sigma_{0}$ and $r_{\mathrm{c}}$ (see Ebeling et al. 1996 for the details of this procedure), and the true total source count rate can be determined from

$s_{\text {true }}=2 \pi \int_{0}^{\infty} \sigma_{\mathrm{K}}(r) r \mathrm{~d} r=\frac{\pi \sigma_{0} r_{\mathrm{c}}^{2}}{3(\beta-1 / 2)}$.

In order to test this flux correction technique on the extended sources found in ACIS fields I perform Monte Carlo simulations of ACIS fields. By using again the package MARX I simulate more than 500 extended sources with a King profile surface brightness convolved with the instrumental PSF. Then, the simulated sources are added to a representative background of Poissonian noise. An ensemble of sources are made for different sets of typical intrinsic parameters (extent, flux, off-axis angle) to determine the expected scatter in the VTP estimates. In Figs. 10-13 results are presented across a large range of source parameters, from extents of $\sim 2$ to 80 arcsec and effective fluxes of $\sim 2 \times 10^{-15}$ to $\sim 1 \times 10^{-13} \mathrm{erg} \mathrm{s}^{-1} \mathrm{~cm}^{-2}$. In all plots, the ratio of 


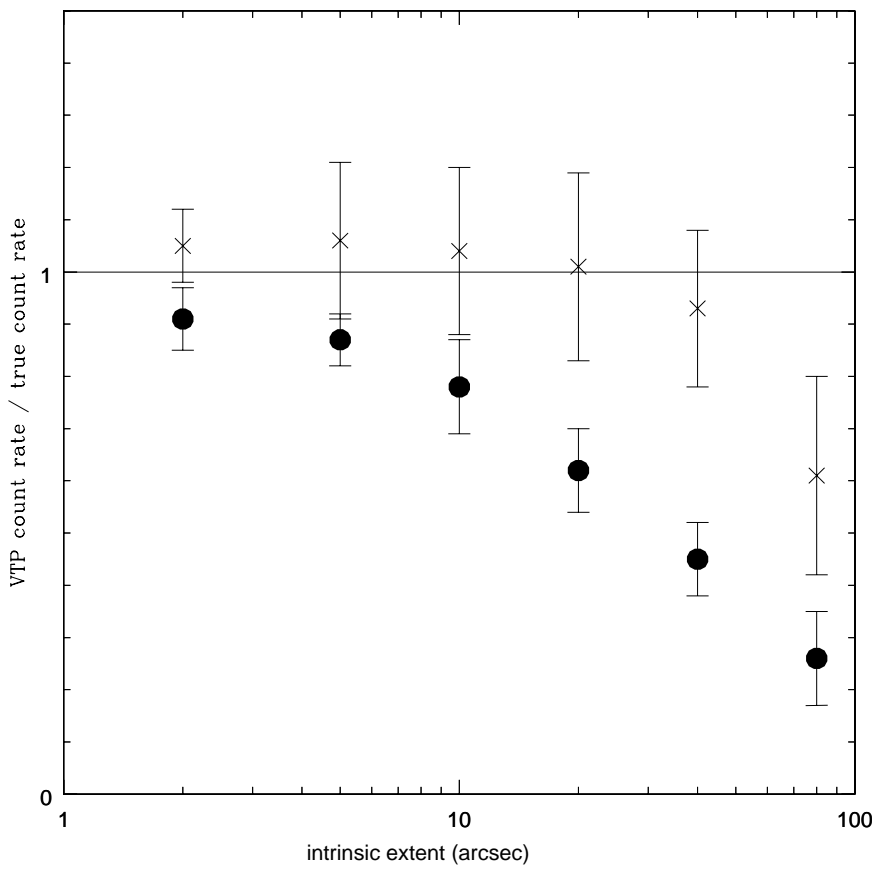

Fig. 10. Mean ratios of detected (filled circles) and corrected (crosses) count rates to the true count rate of high $S N R(>8)$ simulated sources plotted against their extent $(\operatorname{arcsec})$.

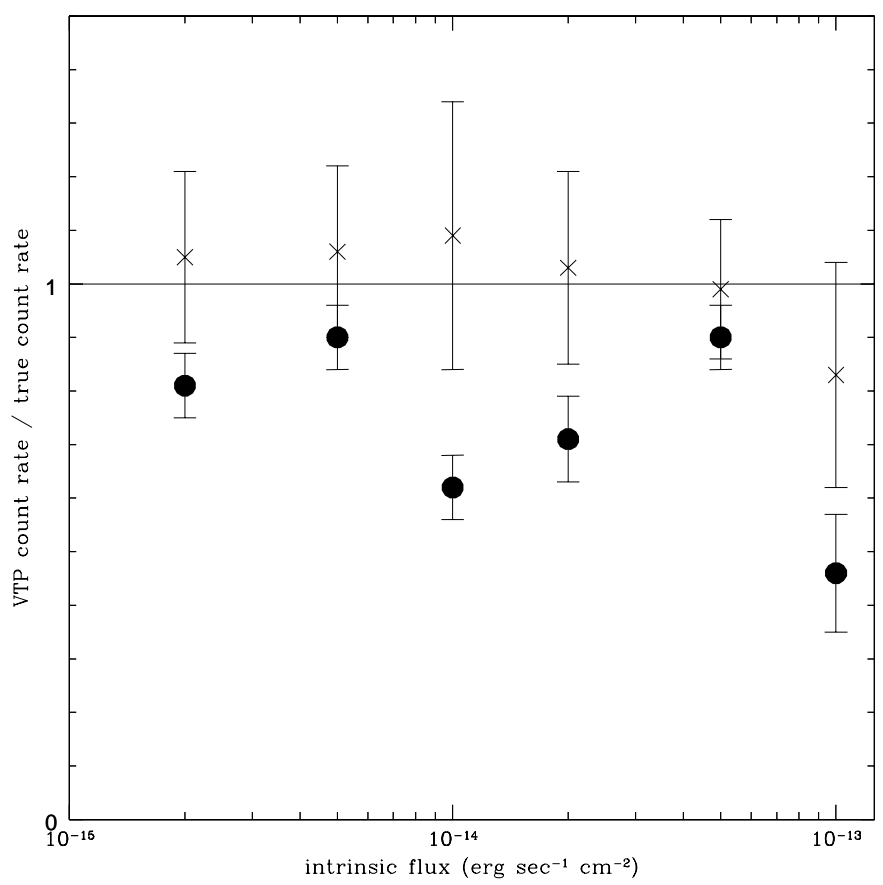

Fig. 11. Mean ratios of detected (filled circles) and corrected (crosses) count rates to the true count rate of high $S N R(>8)$ simulated sources plotted against their intrinsic flux (c.g.s. units).

the raw, detected count rates to the true count rates and the ratio of the corrected (see above) count rates to the true count rate are plotted on the $y$-axes. The effective VTP-detected $S / N$ of a source is a good indicator of the reliability of any flux estimate. As expected, the efficiency of this technique to recover the true count rate of the sources depends significatively on their $S N R$. Since the median signal-to-noise ratio of the real X-ray source

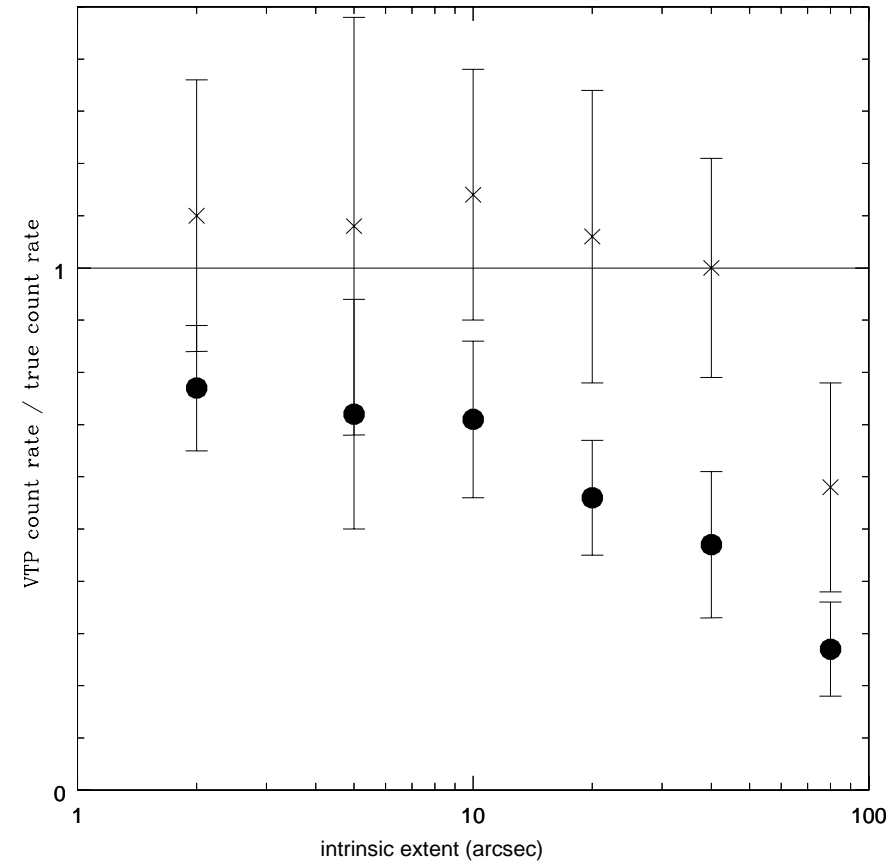

Fig. 12. Mean ratios of detected (filled circles) and corrected (crosses) count rates to the true count rate of high $S N R(<8)$ simulated sources plotted against their extent ( $\operatorname{arcsec})$.

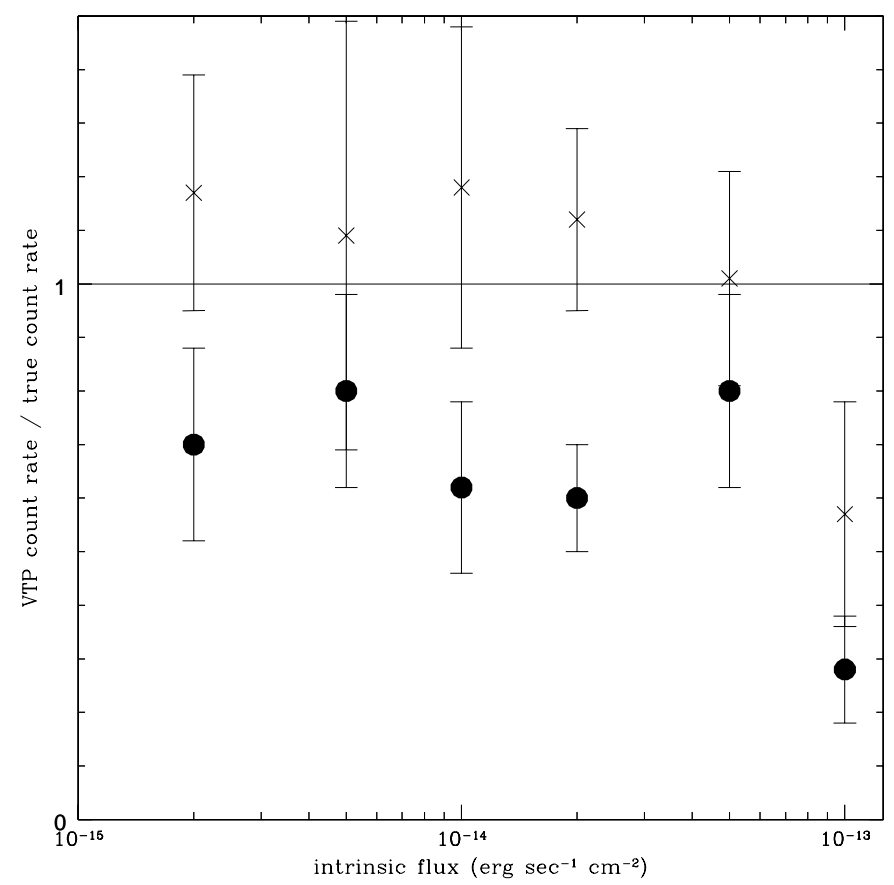

Fig. 13. Mean ratios of detected (filled circles) and corrected (crosses) count rates to the true count rate of high $S N R(<8)$ simulated sources plotted against their intrinsic flux (c.g.s. units).

detection is 8 , I use this to divide the simulation results for presentation. The effect of noise is apparent in the larger scatter seen in flux estimates for the low $S / N(<8)$ sources plotted in Figs. 12 and 13 when compared to those of the high $S / N$ objects in Figs. 10 and 11. For sources of medium to small extent (i.e. $<40$ arcsec) with $S / N$ greater than 8 , I can recover the true flux to whitin $10 \%$ over the flux range shown here. For sources 
of larger extent (of which I might expect to see very few in this survey), the fluxes are systematically underestimated, as expected, since most of the flux now lies below the surface brightness limits. For the low $S / N$ sources $(S / N<8)$, the same general trends are present but are dominated by the scatter caused by noise. For small extents ( $<40$ arcsec) I can still recover the true flux of the faintest objects to within $10 \%-20 \%$.

\subsection{Computation of source fluxes}

The ACIS field experiences non uniform exposure. In ACIS-I observations, for instance, it can vary by as much as $20 \%-25 \%$ from the field center to 10 arcmin radius off-axis. Therefore, exposure maps are built for each pointing (with $4 \times 4$ arcsec resolution pixels) using standard CIAO threads in order to take into account this effect when computing source count rates.

After the count rate correction procedure, source count rates are converted into fluxes multiplying them by a factor which depends on the global characteristics of the telescopedetector system, on the value of the line-of-sight neutral hydrogen column density, on the energy spectrum of the source and on its redshift. I compute this conversion factor by assuming that the extended sources exibit a thermal Raymond-Smith (1977) spectrum with temperature $k T=5 \mathrm{keV}$ and solar abundance ratio 0.4 . For a given hydrogen column density, this conversion factor is accurate to within $8 \%$ for an ICM temperature in the range $1-20 \mathrm{keV}$ and a solar abundance ratio in the range $0.2-1$. The hydrogen column density along the line of sight of the sources is taken from Dickey \& Lockman (1990). Redshifts are not known for most of the candidate clusters at this stage of the work, therefore the fluxes listed in the cluster catalog (see next section) should be considered as provisional.

Finally, I perform a first optical follow-up of the extended sources by analyzing the corresponding $R$ band fields in the Second STScI Digitized Sky Survey (DSS-II, e.g. McLean et al. 2000). These optical fields are sufficiently deep to allow me to identify low redshift X-ray bright galaxies (typically spiral galaxies) that appear as extended sources in the X-ray image. Of the 51 detected extended sources, 15 are identified as single galaxies. Therefore, these sources are excluded from the final catalogue of candidate clusters (as an example see Fig. 14).

\section{The catalogue of candidate clusters}

This survey currently covers a total sky area of $5.55 \mathrm{deg}^{2}$. Using the VTP method, the sample of candidate clusters contains 36 objects with detected flux greater than $1 \times$ $10^{-15} \mathrm{erg} \mathrm{s}^{-1} \mathrm{~cm}^{-2}$. In table $1 \mathrm{I}$ list the detected candidates in growing order of right ascension. The object number is given in Col. 1. The equatorial coordinates (J2000.0) of the X-ray centroid are listed in Cols. 2 and 3 in degrees with four decimals (typical centroid uncertainties are of 1-2 arcsec). The total unabsorbed X-ray flux in c.g.s. units $\left(\mathrm{erg} \mathrm{s}^{-1} \mathrm{~cm}^{-2}\right)$ and its $1 \sigma$ uncertainty are listed in Cols. 4 and 5. The estimated characteristic radius $r_{\mathrm{VTP}}$ is given in Col. 6. Column 7 lists the signal-tonoise ratio of the sources (as defined in Sect. 5). Finally, Col. 8 contains notes for individual clusters. In this column, I also

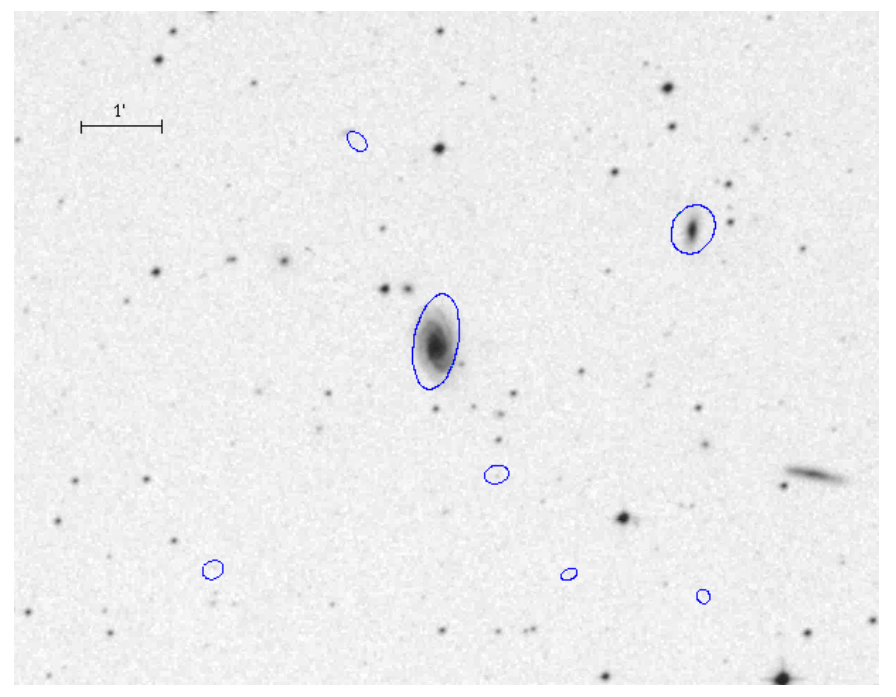

Fig. 14. This figure represents an $R$-band DSS-II image of an ACIS-I field. The ellipses represent the sources detected by VTP in the corresponding X-ray image, two of which are clearly extended. The image shows they correspond to relatively nearby galaxies. Therefore, these extended sources are not listed in the catalogue of candidate clusters.

list the identifications from the literature. Only five candidates seem associated in DSS-II images to galaxy concentrations with respect to the background/foreground galaxy distribution (see Fig. 15) and are probably low-redshift systems. Three candidates are re-discoveries of systems at low-intermediate redshift detected in previous X-ray surveys. In most cases no optical counterpart (galaxy, group, etc.) is seen. This suggests that most candidate clusters are probably at redshifts $z>0.2-0.3$, thus invisible in the relatively shallow fields of the DSS-II.

\section{Sky coverage of the survey}

As already pointed out in the introduction, the aim of a new $\mathrm{X}$-ray selected cluster catalogue is twofold: providing a set of targets for optical/NIR follow-up observations of single interesting objects and to address global statistical properties of the cluster population useful for cosmological studies. This second purpose requires the evalution of the statistical properties of the survey sample (e.g. the $\log N-\log S$ and the luminosity functions). To do this, the knowledge of the effective sky coverage, i.e. the survey area in which all sources above a given flux are detected, is necessary.

Since this survey uses pointed data, each field has a different detection sensitivity to objects of a given extent and flux, according to exposure and background. In order to estimate this, VTP's detection sensitivity can be measured parametrizing a detection criterion based on a definition of the source signalto-noise ratio. In particular, from simulations and real data I find that the criterion for source detection is approximable as

$S N R \equiv \frac{n_{\mathrm{VTP}}-n_{\mathrm{bkg}}}{\sqrt{n_{\mathrm{VTP}}}}>3$,

where $n_{\mathrm{VTP}}$ is the total number of photons (source and background) that lie within the equivalent source radius $r_{\mathrm{VTP}}=$ $\left(A_{\mathrm{VTP}} / \pi\right)^{1 / 2}$, where $A_{\mathrm{VTP}}$ is, as previously defined, the source area 
Table 1. The cluster catalogue.

\begin{tabular}{|c|c|c|c|c|c|c|c|}
\hline Number & $\begin{array}{c}\text { RA }(\mathrm{J} 2000) \\
\text { degrees } \\
(2)\end{array}$ & $\begin{array}{c}\text { Dec (J2000) } \\
\text { degrees } \\
(3)\end{array}$ & $\begin{array}{c}F_{\mathrm{X}} \\
\text { (c.g.s. units) } \\
\text { (4) }\end{array}$ & $\begin{array}{c}\delta F_{\mathrm{X}} \\
\text { (c.g.s. units) } \\
(5)\end{array}$ & $\begin{array}{c}r_{\mathrm{VTP}} \\
(\operatorname{arcsec}) \\
(6)\end{array}$ & $S N R$ & Note \\
\hline $1 \ldots \ldots \ldots . .$. & 7.7555 & +26.4441 & $2.1 \times 10^{-14}$ & $0.3 \times 10^{-14}$ & 26.2 & 7.73 & \\
\hline $2 \ldots \ldots \ldots \ldots$ & 24.1793 & +20.8143 & $1.0 \times 10^{-14}$ & $0.2 \times 10^{-14}$ & 29.6 & 6.85 & $\mathrm{P}$ \\
\hline 3.............. & 44.3881 & -23.4017 & $0.3 \times 10^{-14}$ & $0.1 \times 10^{-14}$ & 17.7 & 2.91 & \\
\hline $4 \ldots \ldots \ldots .$. & 46.4686 & +03.8439 & $0.8 \times 10^{-14}$ & $0.3 \times 10^{-14}$ & 21.3 & 3.82 & \\
\hline $5 \ldots \ldots \ldots \ldots$ & 54.4602 & -23.0730 & $2.4 \times 10^{-14}$ & $0.3 \times 10^{-14}$ & 27.2 & 9.52 & \\
\hline $6 \ldots \ldots \ldots . .$. & 73.5050 & -03.1444 & $2.0 \times 10^{-14}$ & $0.3 \times 10^{-14}$ & 27.3 & 6.42 & \\
\hline 7............... & 73.6638 & -03.1277 & $0.7 \times 10^{-14}$ & $0.2 \times 10^{-14}$ & 16.6 & 3.84 & \\
\hline $8 \ldots \ldots \ldots . .$. & 73.7697 & -10.2003 & $3.2 \times 10^{-14}$ & $0.3 \times 10^{-14}$ & 49.3 & 10.95 & \\
\hline $9 \ldots \ldots \ldots \ldots$ & 73.7895 & -10.1752 & $1.4 \times 10^{-14}$ & $0.2 \times 10^{-14}$ & 37.5 & 6.71 & \\
\hline $10 \ldots \ldots \ldots$ & 85.6665 & -40.9155 & $0.5 \times 10^{-14}$ & $0.1 \times 10^{-14}$ & 14.7 & 6.63 & $\mathrm{P}$ \\
\hline $11 \ldots \ldots \ldots$ & 125.0098 & +63.7611 & $1.7 \times 10^{-14}$ & $0.3 \times 10^{-14}$ & 30.2 & 5.79 & \\
\hline $12 \ldots \ldots \ldots$ & 133.0950 & +51.3794 & $2.1 \times 10^{-14}$ & $0.2 \times 10^{-14}$ & 36.4 & 13.45 & \\
\hline $13 \ldots \ldots \ldots$ & 133.2558 & +51.2218 & $1.2 \times 10^{-14}$ & $0.1 \times 10^{-14}$ & 26.1 & 11.33 & B \\
\hline $14 \ldots \ldots \ldots$ & 137.5350 & +54.3149 & $1.4 \times 10^{-14}$ & $0.1 \times 10^{-14}$ & 27.4 & 15.45 & \\
\hline $15 \ldots \ldots \ldots$ & 142.7633 & +79.2219 & $3.0 \times 10^{-13}$ & $0.1 \times 10^{-13}$ & 75.5 & 29.45 & \\
\hline $16 \ldots \ldots \ldots$ & 148.6100 & +68.9119 & $0.7 \times 10^{-14}$ & $0.1 \times 10^{-14}$ & 26.5 & 6.37 & B \\
\hline $17 \ldots \ldots \ldots$ & 158.8064 & +57.8383 & $1.0 \times 10^{-14}$ & $0.1 \times 10^{-14}$ & 29.3 & 7.74 & \\
\hline $18 \ldots \ldots \ldots$ & 158.8564 & +57.8472 & $1.2 \times 10^{-14}$ & $0.1 \times 10^{-14}$ & 29.1 & 8.69 & \\
\hline $19 \ldots \ldots \ldots$ & 164.0525 & -03.5845 & $5.0 \times 10^{-14}$ & $0.2 \times 10^{-14}$ & 33.7 & 19.27 & $P$ \\
\hline $20 \ldots \ldots \ldots$ & 169.3591 & +07.7274 & $2.6 \times 10^{-14}$ & $0.3 \times 10^{-14}$ & 36.9 & 8.84 & 1 \\
\hline $21 \ldots \ldots \ldots$ & 169.3746 & +07.7716 & $0.9 \times 10^{-14}$ & $0.2 \times 10^{-14}$ & 26.7 & 4.77 & 2 \\
\hline $22 \ldots \ldots \ldots$ & 175.0341 & -26.5263 & $1.5 \times 10^{-14}$ & $0.2 \times 10^{-14}$ & 32.0 & 8.23 & \\
\hline $23 \ldots \ldots \ldots$ & 199.2258 & +29.2394 & $2.4 \times 10^{-15}$ & $0.5 \times 10^{-15}$ & 19.5 & 6.05 & \\
\hline $24 \ldots \ldots \ldots$ & 199.3051 & +29.3089 & $1.2 \times 10^{-15}$ & $0.4 \times 10^{-15}$ & 14.9 & 4.14 & \\
\hline $25 \ldots \ldots \ldots$ & 204.4194 & +29.4313 & $2.0 \times 10^{-15}$ & $0.8 \times 10^{-15}$ & 17.3 & 3.14 & \\
\hline $26 \ldots \ldots \ldots$ & 209.8918 & +62.3164 & $3.1 \times 10^{-13}$ & $0.6 \times 10^{-14}$ & 69.4 & 49.72 & 3 \\
\hline $27 \ldots \ldots \ldots$ & 218.1304 & -01.1376 & $1.4 \times 10^{-14}$ & $0.3 \times 10^{-14}$ & 26.4 & 5.82 & \\
\hline $28 \ldots \ldots \ldots$ & 233.5057 & +23.6009 & $1.1 \times 10^{-14}$ & $0.1 \times 10^{-14}$ & 19.7 & 8.21 & \\
\hline $29 \ldots \ldots \ldots$ & 234.9979 & -03.0390 & $5.7 \times 10^{-14}$ & $0.3 \times 10^{-14}$ & 51.1 & 19.89 & B \\
\hline $30 \ldots \ldots \ldots$ & 237.3827 & +21.5499 & $3.4 \times 10^{-14}$ & $0.3 \times 10^{-14}$ & 33.4 & 14.15 & \\
\hline $31 \ldots \ldots \ldots$ & 245.9976 & +26.6492 & $0.5 \times 10^{-14}$ & $0.1 \times 10^{-14}$ & 14.5 & 4.50 & \\
\hline $32 \ldots \ldots \ldots$ & 249.1558 & +41.1306 & $0.8 \times 10^{-14}$ & $0.1 \times 10^{-14}$ & 25.6 & 9.85 & \\
\hline $33 \ldots \ldots \ldots$ & 255.7827 & +51.5614 & $0.9 \times 10^{-14}$ & $0.2 \times 10^{-14}$ & 29.3 & 5.08 & B \\
\hline $34 \ldots \ldots \ldots$ & 310.9874 & +77.1801 & $0.7 \times 10^{-14}$ & $0.2 \times 10^{-14}$ & 24.1 & 3.46 & \\
\hline $35 \ldots \ldots \ldots$ & 333.2226 & -22.1602 & $1.1 \times 10^{-14}$ & $0.2 \times 10^{-14}$ & 23.7 & 6.03 & \\
\hline $36 \ldots \ldots \ldots$ & 345.7229 & +08.8118 & $1.3 \times 10^{-15}$ & $0.4 \times 10^{-15}$ & 12.4 & 3.81 & \\
\hline
\end{tabular}

Notes: (1) $z=0.4$ cluster (N. 97 Vikhlinin et al. 1998), (2) $z=0.16$ cluster (RIXOS F258_101, Mason et al. 2000), (3) cluster MS 1358.4+6245 at $z=0.328$, (P) flux uncertain due to the presence of a nearby pointlike source, (B) source very close to the border of the X-ray image.

within which the surface brightness exceeds the VTP surface brightness threshold $\sigma_{\mathrm{VTP}}$, which is defined by the background $\left(\sigma_{\mathrm{bkg}}\right)$ and threshold $(f)$ as $\sigma_{\mathrm{vTP}}=f \sigma_{\mathrm{bkg}}(f \geq 1.0)$. Assuming again for the sources a King profile as in Eq. (3), it is possible to compute the SNR for sources with given fluxes and extents in fields with a given exposure time and background surface brightness (see Scharf et al. 1997 for the details of this procedure). Exposure and background information are available for each of the 81 fields used in this survey, therefore I use them to integrate the sky coverage over the area of each ACIS field (including also the PSF variation with off-axis angle) adopting the criterium introduced above to determine the detection sensitivity. The final result shown in Fig. 16 is the combined sky coverage of all fields used in the survey to the limiting threshold $(f=1.0)^{1}$. In this figure I also plot two curves representing the loci, with varying redshift, of galaxy groups $\left(L_{\mathrm{X}}=1 \times 10^{43} \mathrm{erg} \mathrm{s}^{-1}, r_{\mathrm{c}}=100 \mathrm{kpc}\right.$, dashed line $)$ and galaxy clusters $\left(L_{\mathrm{X}}=1 \times 10^{44} \mathrm{erg} \mathrm{s}^{-1}, r_{\mathrm{c}}=150 \mathrm{kpc}\right.$, solid line). Redshifts therefore increase right-to-left along these curves.

\section{Expected redshift distribution of the detected clusters}

An important issue to discuss when compiling a cluster survey is the estimate of the cluster redshift distribution. This is the comoving volume per unit redshift and solid angle $(\mathrm{d} V / \mathrm{d} z \mathrm{~d} \Omega)$

\footnotetext{
${ }^{1}$ For calculations in this and in the following sections cosmic K-corrections from Jones et al. (1998) are used.
} 

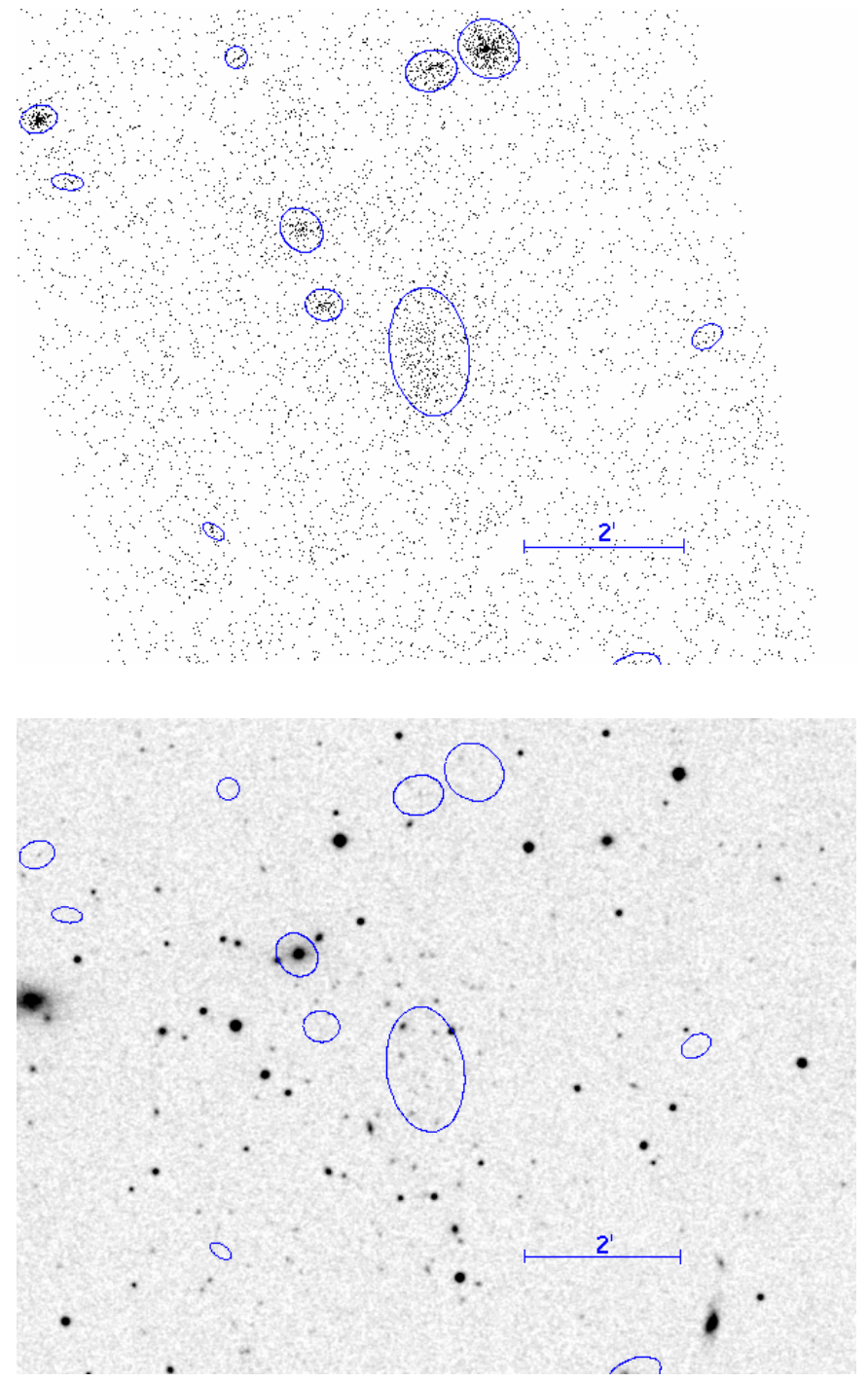

Fig. 15. X-ray and DSS-II $R$ band images of the ACIS-S field containing the candidate cluster 12. Ellipses indicate sources detected by VTP in the X-ray image. The largest one indicates the cluster. Note, in the optical image, the presence of a concentration of faint galaxies in correspondence with the position of the candidate cluster. It suggests the existence of a low redshift system.

times the comoving density of clusters $n_{\text {com }}$ with luminosities above the survey detection $\operatorname{limit} L_{\mathrm{X}}^{\lim }$. I write it as

$$
\frac{\mathrm{d} N}{\mathrm{~d} z \mathrm{~d} \Omega}=\frac{\mathrm{d} V_{\text {com }}}{\mathrm{d} z \mathrm{~d} \Omega} n_{\text {com }}=\frac{\mathrm{d} V_{\text {com }}}{\mathrm{d} z \mathrm{~d} \Omega} \int_{L_{\mathrm{X}}^{\lim }(z)}^{\infty} \mathrm{d} L_{\mathrm{X}} \phi\left(L_{\mathrm{X}}\right),
$$

where $\phi\left(L_{X}\right)$ is the cluster X-ray luminosity function (XLF), i.e. the function describing the number of clusters per unit volume with luminosity in the range $L_{\mathrm{X}} \div L_{\mathrm{X}}+\mathrm{d} L_{\mathrm{X}}$. Now, $\mathrm{d} V_{\text {com }} / \mathrm{d} z \mathrm{~d} \Omega$, the comoving volume per unit solid angle and redshift, is easily computable once a given cosmology is fixed. $L_{X}^{\lim }$ is a function of redshift and depends on the assumed cosmological model, on the shape of the cluster surface brightness profile and on the exposure times of the observations. I compute $L_{X}^{\lim }$ at different redshifts assuming, as in the previous section, a King profile for clusters, a typical exposure time of $40 \mathrm{ks}$ (which is close to the median value of the exposure times of the selected pointings)

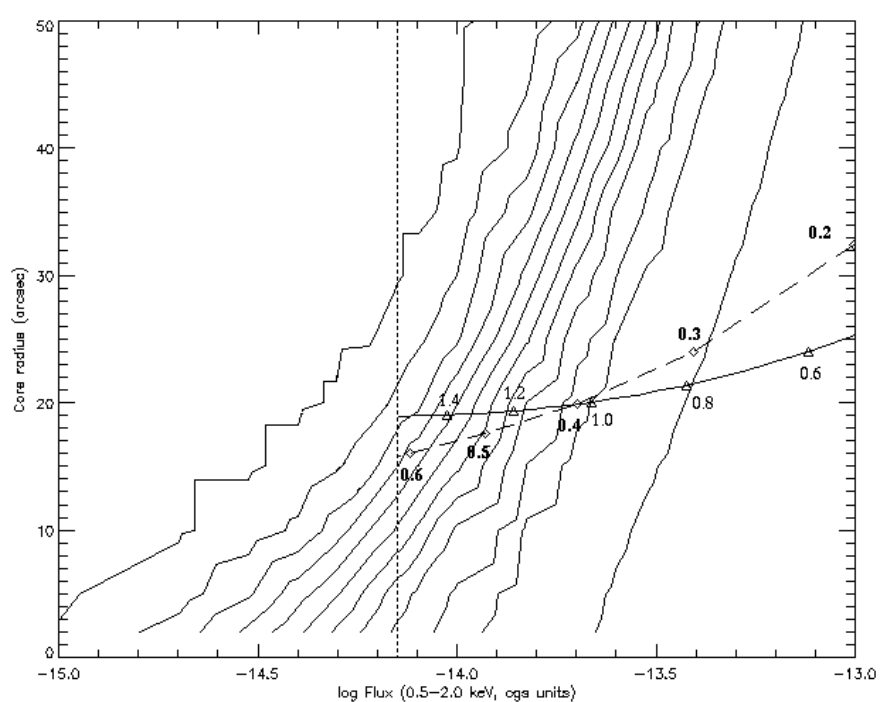

Fig. 16. Sky coverage of the 81 fields used in this survey. Sky coverage is plotted as a function of intrinsic flux and intrinsic, projected core radius (assuming King profile). Contours are drawn at a percentage of the total survey area. From the left, the first contour is at a level of $1 \%$, subsequent solid contours are $10 \%, 20 \%, \ldots, 90 \%, 95 \%$ and $100 \%$. The two curves at the right-hand side of the figure are the loci, with redshift, of galaxy groups $\left(L_{\mathrm{X}}=1 \times 10^{43} \mathrm{erg} \mathrm{s}^{-1}, r_{\mathrm{c}}=100 \mathrm{kpc}\right.$, dashed line) and galaxy clusters $\left(L_{\mathrm{X}}=1 \times 10^{44} \mathrm{erg} \mathrm{s}^{-1}, r_{\mathrm{c}}=150 \mathrm{kpc}\right.$, solid line). Redshifts for these systems increase right to left. The points corresponding to some specific redshift values are marked and labeled on the two curves. The vertical dashed line corresponds to the flux value $7 \times 10^{-15} \mathrm{erg} \mathrm{s}^{-1} \mathrm{~cm}^{-2}$ (see Sect. 7).

and $S N R \geq 3$ as a criterium for cluster detection. For simplicity I also assume a cluster core radius of $150 \mathrm{kpc}$, a quite common value (e.g. Ota \& Mitsuda 2002). Finally I have to integrate the cluster XLF. Following Rosati et al. (2000), I assume it is an evolving Schechter function:

$\phi\left(L_{\mathrm{X}}\right)=\phi_{0}(1+z)^{A} L_{\mathrm{X}}^{-\alpha} \exp \left(-L_{\mathrm{X}} / L_{\mathrm{X}}^{*}\right)$,

with $L_{\mathrm{X}}^{*}=L_{\mathrm{X}, 0}^{*}(1+z)^{B}$, where $A$ and $B$ are two constants parametrizing the possible evolution of the XLF with redshift. For the local XLF, I assume the one derived from the BCS sample (Ebeling et al. 1997). The results are shown in Fig. 17. The three curves represent the expected cluster redshift distribution computed for three different couples of the evolutionary parameters: $A=-0.5$ and $B=-1$ (dotted line), $A=-1$ and $B=-2$ (dashed line), and $A=B=0$ (no evolution, solid line). These curves show that the expected cluster redshift distribution has a maximum at intermediate redshift, at $z \sim 0.3-0.4$. However, a significant fraction of the clusters (about 30-40\% for the two evolutionary models, higher for the no evolution case) are expected to have redshifts higher than $0.6-0.7$, with the possibility of having clusters at $z>1$. The details of this result are dependent on the assumptions done above on cluster profiles. However, I find that changing the cluster profile parameters does not affect the qualitative behaviour of the cluster redshift distribution. Of course, at high redshifts I expect to discover only relatively rich systems, while at low and intermediate redshift the contribution of small galaxy groups becomes significant. This is evident from Fig. 18, where the 


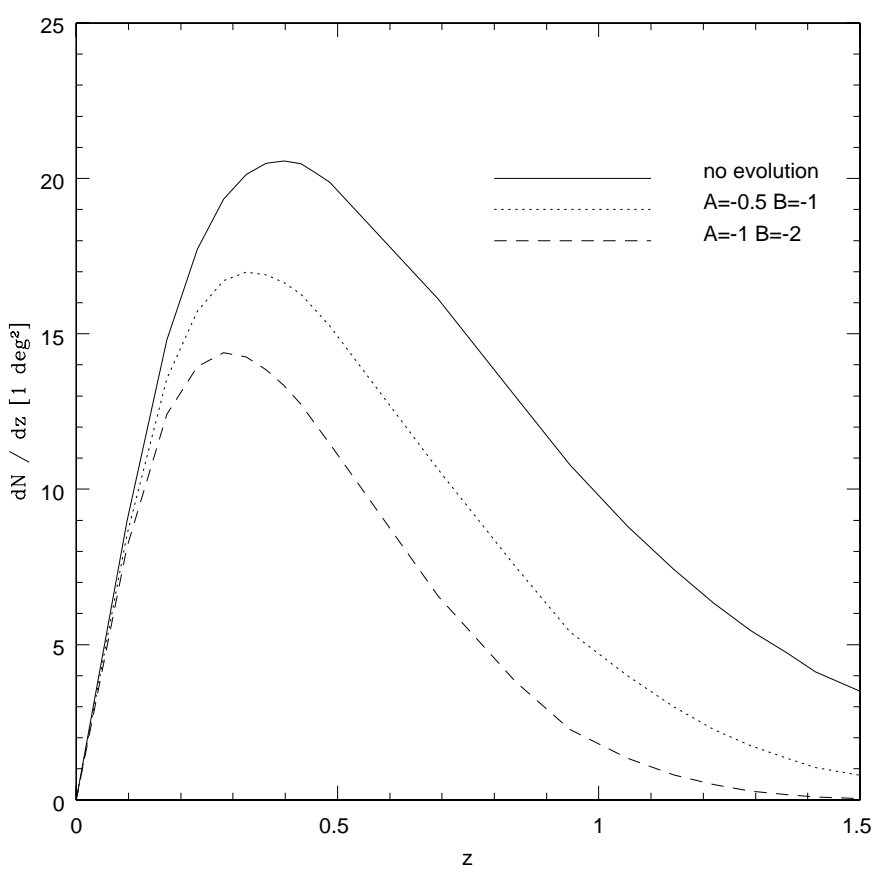

Fig. 17. Expected redshift distribution of the clusters detected in this survey in the redshift range $0 \leq z \leq 1.5$. Three curves related to three different couples of the XLF evolutionary parameters (see text) are drawn.

fractional sky coverage is shown as a function of redshift for three classes of objects. I have complete sky coverage for clusters with $L_{\mathrm{X}}=1 \times 10^{44} \mathrm{erg} \mathrm{s}^{-1}$ and core radius $r_{\mathrm{c}}=150$ $\mathrm{kpc}$ out to a redshift $z \sim 0.8$, and $50 \%$ coverage at a redshift $z \sim 1.3-1.4$. Instead, I have a good coverage for groups (or faint, small clusters) of $L_{\mathrm{X}}=1 \times 10^{43} \mathrm{erg} \mathrm{s}^{-1}$ out to $z \sim 0.5$ (50\% coverage). Galaxies have a $\geq 50 \%$ coverage out to $z \sim 0.2-0.3$. Therefore, galaxies observed in Chandra fields are generally low redshift objects. They can be quite easily recognized in the fields of the Digitized Sky Survey and, eventually, excluded from the catalogue of extended sources, as explained in Sect. 3.4.

The knowledge of the sky coverage also allows to compute the volume that the survey probes above a given redshift $z$, for a given X-ray luminosity, e.g. the characteristic luminosity $L_{\mathrm{X}}^{*}$ corresponding to the "knee" of the XLF. Rosati et al. (2002) compute the search volumes of four cluster surveys: EMSS (Gioia et al. 1990), $160 \mathrm{deg}^{2}$ survey (Vikhlinin et al. 1998), NEP survey (Henry et al. 2001) and RDCS (Rosati et al. 1998). In Fig. 19 I plot the volume probed by these four surveys as a function of redshift together with the volume probed by this survey. Since these surveys cover different solid angles at varying fluxes, they probe different volumes at increasing redshift and therefore different ranges in X-ray luminosities at varying redshifts. From the plot we see that the EMSS is very sensitive to the most luminous systems, but is able to find only a few clusters at high redshifts due to its bright flux limit. NEP, $160 \mathrm{deg}^{2}$ and RDCS are much deeper surveys and discover many clusters at high redshifts. In particular, the RDCS pushes the search to the faintest fluxes, providing sensitivity to the highest redshift systems with characteristic luminosity $L_{\mathrm{X}}^{*}$

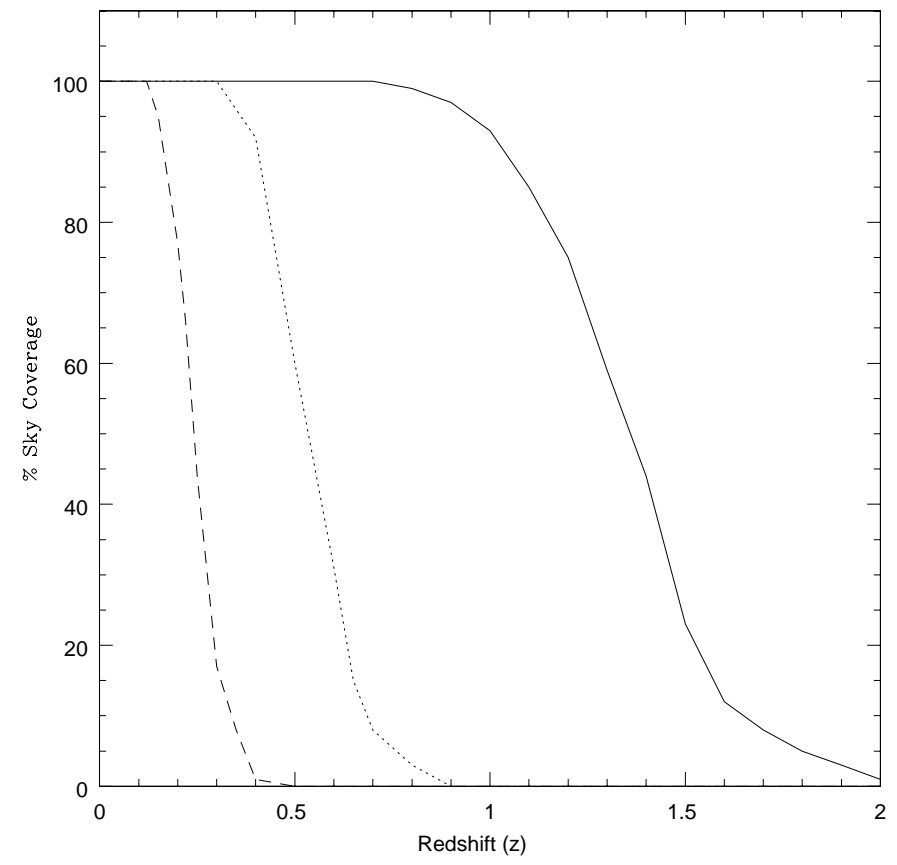

Fig. 18. Sky coverage as a function of redshift for three classes of objects: elliptical galaxies (with $L_{\mathrm{X}}=1 \times 10^{42} \mathrm{erg} \mathrm{s}^{-1}$ and effective core radius $\left.r_{\mathrm{c}}=30 \mathrm{kpc}\right)$ in dashed line, groups $\left(L_{\mathrm{X}}=1 \times 10^{43} \mathrm{erg} \mathrm{s}^{-1}\right.$, $\left.r_{\mathrm{c}}=100 \mathrm{kpc}\right)$ in dotted line, and clusters $\left(L_{\mathrm{X}}=1 \times 10^{44} \mathrm{erg} \mathrm{s}^{-1}\right.$, $r_{\mathrm{c}}=150 \mathrm{kpc}$ ) in solid line.

even beyond $z=1$. Instead, the NEP and the $160 \mathrm{deg}^{2}$ surveys do not reach the flux limit of RDCS and probe the bright end of the XLF out to $z \simeq 1$. This survey (the thick solid line in the figure) probes a small total volume with respect to the other surveys bacause of the still limited surveyed solid angle. Neverthless, it is superior to NEP and $160 \mathrm{deg}^{2}$ surveys in probing the Universe at $z \gtrsim 0.8$ and competitive with RDCS for redshifts $z \gtrsim 1$.

\section{7. $\log N-\log S$ relation for the candidate clusters}

The computed sky coverage of the survey is useful to derive the statistical properties of the cluster population. In particular, it is interesting to calculate the cumulative number density of the candidate clusters as a function of flux (the so-called $\log N$ $\log S$ relation) and compare it with previous studies. In order to perform this calculation, each cluster is added to the cumulative distribution with the weight equal to the inverse solid angle corresponding to its measured flux and angular core radius. The derivative cumulative $\log N-\log S$ function is shown in Fig. 20. Counts are shown as filled circles. The error bars represent the $1 \sigma$ uncertainties in the number of detected clusters. Shown on abscissa is the total source flux, where "total flux" refers to the flux extrapolated to surface brightnesses below the detection limit (see Sect. 3.3). I also show the cluster counts derived from other two deep surveys: The RDCS (Rosati et al. 1995, crosses) and an ultra deep UK ROSAT survey (McHardy et al. 1998, asterisk). The $\log N-\log S$ relation derived from this survey spans a flux range from $7 \times 10^{-15} \mathrm{erg} \mathrm{s}^{-1} \mathrm{~cm}^{-2}$ to $3 \times 10^{-14} \mathrm{erg} \mathrm{s}^{-1} \mathrm{~cm}^{-2}$. The lower flux limit is set at a level in which the sky coverage is about $1 \mathrm{deg}^{2}$ and all clusters are 


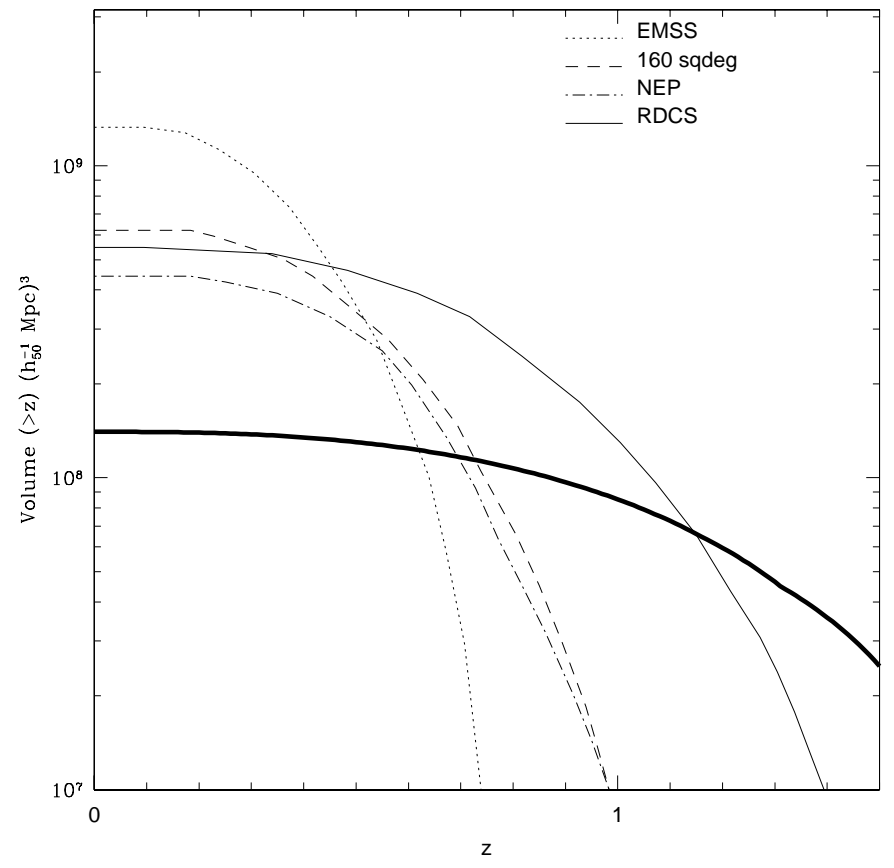

Fig. 19. Search volumes of five $\mathrm{X}$-ray cluster surveys, $V(>\mathrm{z})$, for a cluster of given X-ray luminosity $\left(L_{\mathrm{X}}=3 \times 10^{44} h_{50}^{-2} \mathrm{erg} \mathrm{s}^{-1} \simeq L_{\mathrm{X}}^{*}\right)$. The thick solid line corresponds to the volume probed by this survey (adapted from Rosati et al. 2002).

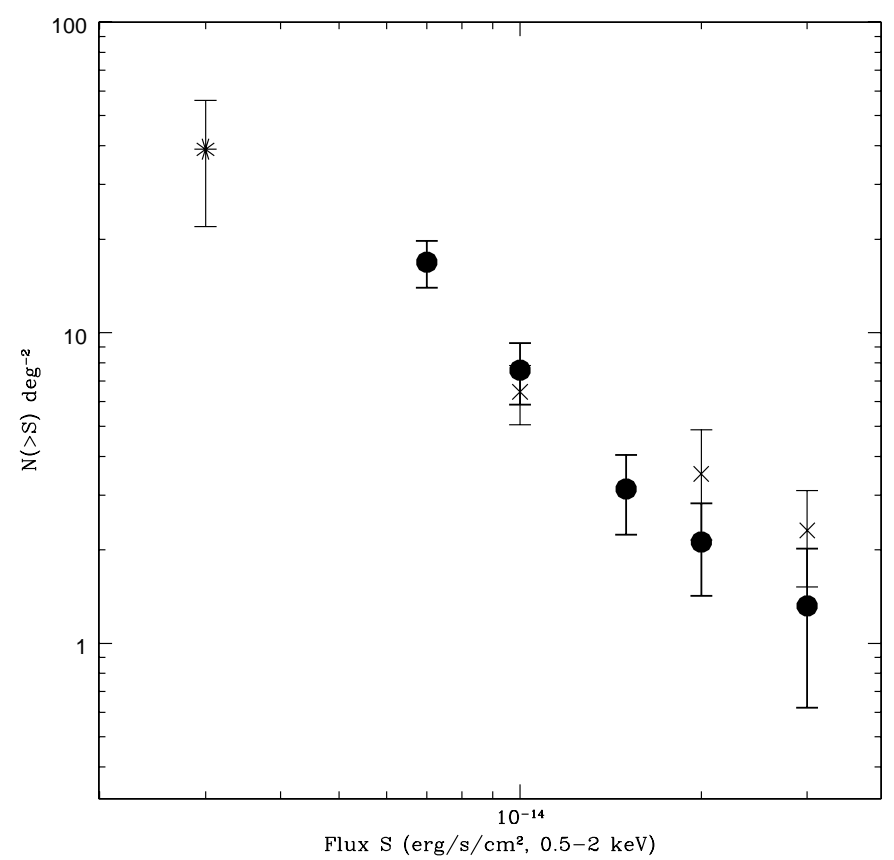

Fig. 20. Cumulative $\log N-\log S$ function of the candidate clusters. Counts are shown as filled circles. The error bars represent the $1 \sigma$ uncertainties in the number of detected clusters. Data from Rosati et al. (1995, crosses) and from McHardy et al. (1998, asterisk) are also shown for comparison.

expected to be detected as extended sources since they have core radii larger than about 15 arcsec, based on a range of typical sizes and luminosities (see Fig. 16). Therefore, I expect this sample be complete to this flux level. At the bright end, the results are in agreement within errors with the deep sample from
Rosati et al. (1995). At the faintest end, the extrapolation of the $\log N-\log S$ agrees with the results of McHardy et al. (1998), who identified most of the X-ray sources, regardless of extent, in their ultradeep ROSAT survey.

\section{Summary}

I present a catalogue of 36 candidate clusters detected as extended sources in 81 Chandra ACIS observations covering an angle of $5.55 \mathrm{deg}^{2}$. To detect these sources, I use a detection algorithm based on the Voronoi Tessellation and Percolation technique. This is an efficient method in order to detect low surface brightness extended sources in a shape independent way. Despite the small solid angle covered so far, the survey probes a volume at high redshift $(z \gtrsim 0.8)$ comparable or larger than previous Einstein and ROSAT-based surveys. Moreover, I expect a significative fraction of the candidates are medium-high redshift $(z>0.6-0.7)$ systems. This makes these candidate clusters interesting targets for optical/NIR follow-up observations. In particular, the confirmed distant clusters will be useful, for instance, to study the properties of the cluster galaxy population at high redshift (e.g. Gioia et al. 1999; Stanford et al. 2002). I also present a preliminary $\log N-\log S$ relation derived from the catalogue. This relation shows a general agreement with some deep ROSAT-PSPC based cluster surveys. As soon as new data will be available in the Chandra archive, they will be reduced in order to increase the sky area and the volume probed by the survey and enrich the catalogue compiled so far.

Acknowledgements. The data used in this work have been obtained from the Chandra data archive at the NASA Chandra X-ray center (http://asc.harvard.edu/cda/) and from the On-line Digitized Sky Survey server at ESO/ST-ECF Archive (http://arch-http.hq.eso.org/dss/dss).

I wish to thank Andrea Biviano, Stefano Borgani, Marisa Girardi and Massimo Ramella for useful suggestions and discussions. I am particularly grateful to Paolo Tozzi for having introduced me in the art of X-ray data reduction.

Work partially supported by the Italian Ministry of Education, University, and Research (MIUR, grant Progetto Giovani 2001, grant COFIN2001028932 "Clusters and groups of galaxies, the interplay of dark and baryonic matter"), and by the Italian Space Agency (ASI).

\section{References}

Arnaud, M., Majerowicz, S., Lumb, D., et al. 2002, A\&A, 390, 27

Bahcall, N. A., \& Cen, R. 1993, ApJ, 407, 49

Borgani, S., \& Guzzo, L. 2001, Nature, 409, 39

Borgani, S., Rosati, P., Tozzi, P., et al. 2001, ApJ, 561, 13

Cavaliere, A., \& Fusco Femiano, R. 1976, A\&A, 49, 137

Chandra X-ray Center Team 2001, The Chandra Proposers' Observatory Guide, version 4.0

Collins, C. A., Guzzo, L., Böhringer, H., et al. 2000, MNRAS, 319, 939

Dickey, J. M., \& Lockman, F. J. 1990, ARA\&A, 28, 215

Dressler, A., Oemler, A. Jr., Couch, W. J., et al. 1997, ApJ, 490, 577

Ebeling, H. 1993, MPE report 250 (ISSN 0718-0719)

Ebeling, H., \& Wiedenmann, G. 1993, Phys. Rev. E, 47, 704

Ebeling, H., Voges, W., Böhringer, H., et al. 1996, MNRAS, 281, 799

Ebeling, H., Edge, A. C., Fabian, A. C., et al. 1997, ApJ, 479, 101 
Ellingson, E., Lin, H., Yee, H. K. C., \& Carlberg, R. G. 2001, ApJ, 547,609

Fruscione, A., \& Siemiginowska, A. 2000, Chandra News, 7, 4

Gioia, I. M., Henry, J. P., Maccacaro, T., et al. 1990, ApJ, 356, 35

Gioia, I. M., Henry, J. P., Mullis, C. R., \& Ebeling, H. 1999, AJ, 117, 2608

Girardi, M., Borgani, S., Giuricin, G., Mardirossian, F., \& Mezzetti, M. 1998, ApJ, 506, 45

Gonzalez, A. H., Zaritsky, D., Dalcanton, J. J., \& Nelson, A. 2001, ApJS, 137, 117

Gonzalez, A. H., Zaritsky, D., \& Wechsler, R. H. 2002, ApJ, 571, 129

Henry, J. P., Gioia, I. M., Mullis, C. R., et al. 2001, ApJ, 553, 109

Holden, B. P., Stanford, S. A., Squires, G. K., et al. 2002, AJ, 124, 33

Jones, C., \& Forman, W. 1984, ApJ, 276, 38

Jones, L. R., Scharf, C., Ebeling, H., et al. 1998, ApJ, 495, 100

Jorgensen, I., Hjorth, J., Franx, M., \& van Dokkum, P. G. 1997, BAAS, 29,780

Kelson, D. D., van Dokkum, P. G., Franx, M., Illingworth, G. D., \& Fabricant, D. 1997, ApJ, 478, 13

Kiang, T. 1966, ZAp, 64, 433

King, I. R. 1962, AJ, 67, 471

Mason, K. O., Carrera, F. J., Hasinger, G., et al. 2000, MNRAS, 311, 456

Mauskopf, P. D., Ade, P. A. R., Allen, S. W., et al. 2000, ApJ, 538, 505

McHardy, I. M., Jones, L. R., Merrifield, M. R., et al. 1998, MNRAS, 295, 641

McLean, B. J., Greene, G. R., Lattanzi, M. G., \& Pirenne, B. 2000, PASP, 216, 145

Nelson, A. E., Gonzalez, A. H., Zaritsky, D., \& Dalcanton, J. J. 2001, ApJ, 563, 629

Nelson, A. E., Gonzalez, A. H., Zaritsky, D., \& Dalcanton, J. J. 2002, ApJ, 566, 103
Nichol, R. C., Collins, C. A., Guzzo, L., \& Lumsden, S. L. 1992, MNRAS, 255, 21

Nonino, M., Bertin, E., da Costa, L., et al. 1999, A\&AS, 137, 51

Ota, N., \& Mitsuda, K. 2002, ApJ, 567, 23

Pointecouteau, E., Hattori, M., Neumann, D., et al. 2002, A\&A, 387, 56

Postman, M., Lubin, L. M., Gunn, J. E., et al. 1996, AJ, 111, 615

Ramella, M., Boschin, W., Fadda, D., \& Nonino, M. 2001, A\&A, 368, 3,776

Raymond, J. C., \& Smith, B. W. 1977, ApJS, 35, 419

Reese, E. D., Mohr, J. J., Carlstrom, J. E., et al. 2000, ApJ, 533, 38

Reiprich, T. H., \& Böhringer, H. 1999, Astr. Nachr., 320, 296

Reiprich, T. H., \& Böhringer, H. 2002, ApJ, 567, 716

Rosati, P., Della Ceca, R., Burg, R., Norman, C., \& Giacconi, R. 1995, ApJ, 445, 11

Rosati, P., Della Ceca, R., Norman, C., \& Giacconi, R. 1998, ApJ, 492, 21

Rosati, P., Borgani, S., Della Ceca, R., et al. 2000, Proceedings of the Workshop Large Scale Structure in the X-ray Universe, 1999, Santorini (Greece)

Rosati, P., Borgani, S., \& Norman, C. 2002, ARA\&A, 40, 539

Scharf, C. A., Jones, L. R., Ebeling, H., et al. 1997, ApJ, 477, 79

Scharf, C. A. 2002, ApJ, 572, 157

Schuecker, P., Böhringer, H., Guzzo, L., et al. 2001, A\&A, 368, 86

Stanford, S. A., Holden, B. P., Rosati, P., et al. 2002, AJ, 123, 619

Sunyaev, R. A., \& Zeldovich, Y. B. 1972, Comm. Astrophys. Space Phys., 4, 173

van Dokkum, P. G., \& Franx, M. 2001, ApJ, 553, 90

Vikhlinin, A., McNamara, B. R., Forman, W., et al. 1998, ApJ, 502, 558

Voronoi, G. 1908, J. Reine Angew. Math., 134, 198

Wise, M. 1997, Chandra News (published as AXAF News), 5, 22 